\title{
Decreased Level of Exosomal miR-5121 Released from Microglia Suppresses Neurite Outgrowth and Synapse Recovery of Neurons Following Traumatic Brain Injury
}

\author{
Chengcheng Zhao ${ }^{1,2} \cdot$ Yuefei Deng ${ }^{3} \cdot \mathrm{Yi} \mathrm{He}^{1} \cdot$ Xianjian Huang ${ }^{1} \cdot$ Chuanfang Wang $^{4} \cdot$ Weiping $\mathrm{Li}^{1}$
}

Accepted: 20 December 2020 / Published online: 21 January 2021

(C) The American Society for Experimental NeuroTherapeutics, Inc. 2021

\begin{abstract}
Activated microglia can suppress neurite outgrowth and synapse recovery in the acute stage following traumatic brain injury (TBI). However, the underlying mechanism has not been clearly elucidated. Exosomes derived from microglia have been reported to play a critical role in microglia-neuron interaction in healthy and pathological brains. Here, we aimed to investigate the role of microglia-derived exosomes in regulating neurite outgrowth and synapse recovery following TBI. In our study, exosomes derived from microglia were co-cultured with stretch-injured neurons in vitro and intravenously injected into mice that underwent fluid percussion injury (FPI) by tail vein injection in vivo. The results showed that microglia-derived exosomes could be absorbed by neurons in vitro and in vivo. Moreover, exosomes derived from stretch-injured microglia decreased the protein levels of GAP43, PSD-95, GluR1, and Synaptophysin and dendritic complexity in stretch-injured neurons in vitro, and reduced GAP43+ NEUN cell percentage and apical dendritic spine density in the pericontusion region in vivo. Motor coordination was also impaired in mice treated with stretch-injured microglia-derived exosomes after FPI. A microRNA microarray showed that the level of miR-5121 was decreased most greatly in exosomes derived from stretch-injured microglia. Overexpression of miR-5121 in stretch-injured microglia-derived exosomes partly reversed the suppression of neurite outgrowth and synapse recovery of neurons both in vitro and in vivo. Moreover, motor coordination in miR-5121 overexpressed exosomes treated mice was significantly improved after FPI. Following mechanistic study demonstrated that miR-5121 might promote neurite outgrowth and synapse recovery by directly targeting RGMa. In conclusion, our finding revealed a novel exosomemediated mechanism of microglia-neuron interaction that suppressed neurite outgrowth and synapse recovery of neurons following TBI.
\end{abstract}

Key Words Exosomes $\cdot$ microRNA $\cdot$ microglia $\cdot$ neuron $\cdot$ traumatic brain injury

$\begin{array}{ll}\text { Abbreviations } \\ \text { BSA } & \text { Bovine serum albumin } \\ \text { CNS } & \text { Central nervous system } \\ \text { FBS } & \text { Fetal bovine serum }\end{array}$

Chengcheng Zhao, Yuefei Deng and Yi He contributed equally to this work.

\section{Chuanfang Wang} cfwang2007@126.com

Weiping Li wpli@szu.edu.cn

1 Department of Neurosurgery, The First Affiliated Hospital of Shenzhen University, Shenzhen Second People's Hospital, 3002 Sungang Road, Shenzhen, Guangdong, China
FPI Fluid percussion injury

GAP43 Growth-associated protein 43

GAPDH Glyceraldehyde 3-phosphate dehydrogenase

GluR1 Glutamate receptor 1
2 Guangdong Key Laboratory for Biomedical Measurements and Ultrasound Imaging, School of Biomedical Engineering, Shenzhen University Health Science Center, Shenzhen, Guangdong, China

3 Department of Neurosurgery, Sun Yat-sen Memorial Hospital, Sun Yat-sen University, Guangzhou, Guangdong, China

4 Department of Neurosurgery and Neurosurgical Disease Research Centre, The Second Affiliated Hospital of Guangzhou Medical University, 250 Changgang East Road, Guangzhou, Guangdong, China 


$\begin{array}{ll}\text { IL-1 } \beta & \text { Interleukin-1 } \beta \\ \text { IL-6 } & \text { Interleukin-6 } \\ \text { IL-10 } & \text { Interleukin-10 } \\ \text { iNOS } & \text { Inducible nitric oxide synthase } \\ \text { PBS } & \text { Phosphate-buffered saline } \\ \text { PFA } & \text { Paraformaldehyde } \\ \text { PSD-95 } & \text { Post-synaptic density-95 } \\ \text { RGMa } & \text { Repulsive guidance molecule family member A } \\ \text { RhoA } & \text { Ras homolog family member A } \\ \text { TBI } & \text { Traumatic brain injury } \\ \text { TEM } & \text { Transmission electron microscopy }\end{array}$

\section{Introduction}

Traumatic brain injury (TBI) is a leading cause of injuryinduced neurological disability and death worldwide $[1,2]$. Although rapid advances in the understanding of pathophysiology of TBI were obtained, patients with moderate to severe TBI still suffer from long-term neurological deficits [3, 4]. Therefore, how to improve neurological function recovery after TBI remains to be explored.

There is evidence that TBI induces axonal transection and synapse loss which cause neurological function deficits $[5,6]$. Strategies that enhance the intrinsic regeneration capacity of injured neurons and ameliorate the detrimental microenvironment for neurite regeneration and synapse recovery can significantly restore neurological function [7-9].

Microglia are resident mononuclear phagocytes and account for $10-15 \%$ of all cells in the central nervous system (CNS) [10]. In physiological condition, microglia play a critical role in maintaining CNS homeostasis, synaptic plasticity, axon outgrowth, and neuronal death [11-13]. After TBI, microglia converge onto the damage site within minutes and undergo a time-dependent and injury-associated transcriptomic profile changes $[14,15]$. In the acute stage following TBI, the microglial transcriptomic profile changes towards a pro-inflammatory dominant type which may aggravate cell death and impede neurite regeneration and synapse recovery [15-17]. However, the underlying mechanism is not entirely understood and remains to be elucidated.

Recent study has focused on the function of exosomes secreted by microglia. Exosomes are small membrane vesicles $(40-150 \mathrm{~nm}$ in diameter) released into extracellular space by cells. These vesicles are originated from multivesicular bodies by invagination of the membrane of endosomal vesicles [18]. Exosomes can transfer proteins, lipids, and nucleic acids between cells via endocytosis, and thus they may be associated with cell to cell communication [19]. Evidence has suggested that microglia-derived exosomes may play a critical role in neurite outgrowth after TBI [20]. Therefore, we hypothesized that activated microglia may suppress neurite outgrowth and synapse recovery partially through microglia-derived exosomes in the acute stage following TBI. To test this hypothesis, we utilized an in vitro cell stretch injury model and an in vivo moderate fluid percussion injury model to investigate the impact of TBI on exosomal microRNAs derived from microglia and the role of microglial exosomal microRNAs on injured neurons. We evaluated neurite outgrowth and synapse recovery of injured neurons in vitro and in vivo after the neurons took up microglia-derived exosomes. Our findings indicate that activated microglia may suppress neurite outgrowth and synapse recovery partially through downregulating exosomal miR-5121 after TBI.

\section{Materials and Methods}

\section{BV2 Cell Culture, Stretch-Induced Injury, and LPS Stimulation}

BV2 microglial cells were cultured in DMEM-High Glucose Medium (Hyclone, Logan, UT, USA) with 10\% fetal bovine serum (FBS, Gibco, Grand Island, NY, USA).

For stretch-induced injury, BV2 microglial cells were seeded into six-well Bioflex plates (Flexcell, Hillsborough, NC, USA) pre-coated with poly-D-lysine (Sigma-Aldrich, St. Louis, MO, USA) at a density of $5 \times 10^{5}$ cells $/$ well. Before stretch-induced injury, BV2 cells were washed twice with phosphate-buffered saline (PBS) and cultured in DMEMHigh Glucose Medium without FBS. BV2 cells were then subjected to stretch-induced injury by cell injury controller II (Custom Design and Fabrication, USA) according to the manufacturer's instructions [21, 22]. In brief, the six-well Bioflex plate was placed into the culture tray holder and a pressure plug was firmly placed over the well. The delay was set to $50 \mathrm{~ms}$ and the regulator pressure was set to $20 \pm 5 \mathrm{psi}$ for mild stretch-induced injury and $40 \pm 5$ psi for severe stretchinduced injury. Stretch-induced injury of BV2 cells was then produced by a pulse of compressed nitrogen gas, which deformed the silastic membrane and adherent BV2 cells. The peak pressure to the well was recorded to evaluate the degree of injury. The well with more than 2.0 psi or less than $1.8 \mathrm{psi}$ peak pressure was discarded for mild stretch-induced injury and the well with more than 4.5 psi or less than 3.5 psi peak pressure was discarded for severe stretch-induced injury.

For LPS stimulation, BV2 microglial cells were seeded into six-well culture plates at a density of $5 \times 10^{5}$ cells/well. Cells were further treated with $10 \mu \mathrm{g} / \mathrm{mL}$ LPS (Sigma-Aldrich, St. Louis, MO, USA) for $6 \mathrm{~h}$. After 6-h stimulation, BV2 cells were washed twice with phosphate-buffered saline (PBS) and cultured in DMEM-High Glucose Medium without FBS. 
Primary Microglia Culture and Stretch-Induced Injury

Primary microglia were isolated from cortices of neonatal (P1P2) C57BL/6 mice as previously described with slight modification [23]. After removing hippocampi and meninges, cortices were digested with $0.25 \%$ trypsin (Gibco, Grand Island, NY, USA) for $30 \mathrm{~min}$ at $37^{\circ} \mathrm{C}$. Cells were then suspended in DMEM-F12 Medium (Gibco, Grand Island, NY, USA) containing $10 \%$ FBS and passed through a $70-\mu$ m nylon filter. The cell suspension was seeded into $75-\mathrm{cm}^{2}$ tissue culture flasks at 4 brains per flask. After reaching confluence, mixed glial cultures were incubated with a diluted trypsin solution (0.25\% trypsin diluted 1:4 in DMEM/F12) for $15 \mathrm{~min}$. An intact layer of astrocyte was detached after incubation and microglia remained attached to the bottom of the flask. Isolated microglia were seeded into six-well Bioflex plates pre-coated with poly-D-lysine at a density of $5 \times 10^{5}$ cells/ well for stretch-induced injury.

\section{Isolation, Identification, and Labeling of Microglia- Derived Exosomes}

Exosomes were isolated from microglial culture supernatant at $24 \mathrm{~h}$ after stretch-induced injury or LPS stimulation as previously described [24]. In brief, the cell supernatant was collected and underwent sequential centrifugation at $300 \times g$ for $10 \mathrm{~min}, 2000 \times \mathrm{g}$ for $10 \mathrm{~min}$, and $10,000 \times \mathrm{g}$ for $30 \mathrm{~min}$ at $4{ }^{\circ} \mathrm{C}$. The supernatant was collected and then passed through a $0.22-\mu \mathrm{m}$ sterilized filter (Millipore, Billerica, MA, USA). Afterwards, the supernatant was centrifuged at $100000 \times \mathrm{g}$ for 70 min at $4{ }^{\circ} \mathrm{C}$. For purification of exosomes, pellets were resuspended with PBS and centrifuged at $100000 \times g$ for $70 \mathrm{~min}$ at $4{ }^{\circ} \mathrm{C}$. Pellets (exosomes) were collected and suspended for further experiments.

For identification of microglia-derived exosomes, a transmission electron microscopy (TEM, JEM-2100F, JEOL, Japan) was used to observe the morphology of exosomes. Sample preparation method for TEM is described previously [24]. In brief, exosomes were re-suspended in $50 \mu \mathrm{l}$ of $2 \%$ paraformaldehyde and $5 \mu \mathrm{l}$ of the exosomes was deposited on Formvar-carbon-coated grids for $20 \mathrm{~min}$. The grids were then washed with PBS and incubated with $50 \mu \mathrm{l}$ of $1 \%$ glutaraldehyde for $5 \mathrm{~min}$. After washing with $100 \mu \mathrm{l}$ of distilled water for eight times ( $2 \mathrm{~min}$ each time), the samples were negative staining with $2 \%$ phosphotungstic acid for $1 \mathrm{~min}$. The concentration and size distribution of exosomes was analyzed by nanoparticle tracking analysis (NTA, Nanosight NS 300, Malvern, UK) according to the manufacturer's manual. Biomarkers of exosomes (Alix, CD63, and CD81) were detected by western blot.

Exosomes were labeled with a PKH26 red fluorescent cell linker kit (Sigma-Aldrich, St. Louis, MO, USA) according to the manufacturer's instructions. In brief, exosomes were re- suspended in $1 \mathrm{ml}$ Diluent $\mathrm{C}$ and incubated with $6 \mu \mathrm{l}$ of PKH26 dye for $5 \mathrm{~min}$. After washing with $2 \mathrm{ml}$ of $10 \%$ bovine serum albumin (BSA) in PBS, the samples were centrifuged at $100000 \times g$ for $70 \mathrm{~min}$ at $4{ }^{\circ} \mathrm{C}$. The pellets (exosomes) were collected and suspended for further experiments.

\section{Primary Cortical Neuron Culture}

Primary neurons were obtained from cortices of embryonic day $18 \mathrm{C} 57 \mathrm{BL} / 6$ mice as previously described with slight modification [25]. In brief, cortices were isolated and cleaned of hippocampi and meninges. After cutting into small pieces, the tissue was digested with $0.25 \%$ trypsin (Gibco, Grand Island, NY, USA) at $37^{\circ} \mathrm{C}$. Cells were then suspended in DMEM-F12 Medium (Gibco, Grand Island, NY, USA) containing $10 \%$ FBS and passed through a $70-\mu \mathrm{m}$ nylon filter. The cell suspension was seeded into six-well Bioflex plates pre-coated with poly-D-lysine. Cells were cultured in Neurobasal Medium (Gibco, Grand Island, NY, USA) with 2\% B27 supplement (Gibco, Grand Island, NY, USA) and $500 \mu \mathrm{M}$ glutamine (Gibco, Grand Island, NY, USA). Cytosine arabinoside (Sigma-Aldrich, St. Louis, MO, USA) was added on day 3 to inhibit glial growth. On day 7, neurons were subjected to stretch-induced injury and then co-cultured with BV2-derived exosomes (approximately $2.5 \times 10^{8}$ particles per well) for $24 \mathrm{~h}$. The neurons were randomly divided into six groups including the CON-NEU group (normal cultured neurons); the SI-NEU group (neurons received stretchinduced injury); the SI-NEU+CON-B-EXO group (neurons received stretch-induced injury and co-cultured with exosomes derived from uninjured BV2); the SI-NEU+SI-BEXO group (neurons received stretch-induced injury and cocultured with exosomes derived from stretch-injured BV2); the SI-NEU+SI-NC-B-EXO group (neurons received stretch-induced injury and co-cultured with exosomes derived from stretch-injured BV2 transduced with empty vector lentivirus); and the SI-NEU+SI-miR-5121 OE-B-EXO group (neurons received stretch-induced injury and co-cultured with exosomes derived from stretch-injured BV2 transduced with miR-5121 overexpression lentivirus).

\section{Cell Viability Assay}

After stretch-induced injury, uninjured BV2 cells and severe stretch-injured BV2 cells $\left(5 \times 10^{3} /\right.$ well $)$ were seeded in 96 well plates and placed in the incubator for $24 \mathrm{~h}$. A cell counting kit-8 (CCK-8) assay (Beyotime, Jiangsu, China) was performed to evaluate cell viability. In brief, after replacement of medium, $10 \mu \mathrm{lCCK}-8$ reagent was added and cells were incubated for $4 \mathrm{~h}$. The optical density was then measured at $450 \mathrm{~nm}$ using a Berthold TriStar ${ }^{2}$ S LB 942 multimode reader. 
Primary neurons $\left(5 \times 10^{3} /\right.$ well $)$ were seeded in 96 -well plates and incubated with different concentration of microglia-derived exosomes $\left(2.5 \times 10^{8}\right.$ particles $/ \mathrm{ml}$ and $2.5 \times 10^{9}$ particles $/ \mathrm{ml}$ ) for $24 \mathrm{~h}$. A CCK- 8 assay was then performed to evaluate cell viability.

\section{Flow Cytometry}

Microglial exosomes derived from uninjured BV2 microglia and stretch-injured BV2 microglia were labeled with the PKH26 red fluorescent cell linker kit and then co-cultured with uninjured neurons and stretch-injured neurons. After $24 \mathrm{~h}$, the neurons were harvested using $0.25 \%$ trypsin and washed twice by PBS. Subsequently, the neurons were acquired by using a Beckman Coulter Navios flow cytometer, and the geometric mean of fluorescence intensity of PKH26 was analyzed using FlowJo VX software.

\section{Animals}

Thy1-GFP knock-in mice were generously provided by Professor Jiyao Jiang (Department of Neurosurgery, Ren Ji Hospital, School of Medicine, Shanghai Jiao Tong University). Animals were housed in the animal facility and maintained at controlled temperature and humidity under standard 12-h light/dark cycles with ad libitum access to water and chow. One hundred and twenty-six adult (8- to 10-week-old) male Thy1-GFP knock-in mice were randomly divided into eight groups including the SHAM group (mice underwent identical surgical procedure without application of lateral fluid percussion injury (FPI), $n=19$ ); the FPI+PBS group (mice underwent FPI and received tail vein injection of PBS, $n=$ 19); the FPI+CON-B-EXO group (mice underwent FPI and received tail vein injection of exosomes derived from uninjured BV2, $n=19$ ); the FPI+SI-B-EXO group (mice underwent FPI and received tail vein injection of exosomes derived from stretch-injured BV2, $n=19$ ); the FPI+SI-NC-BEXO group (mice underwent FPI and received tail vein injection of exosomes derived from stretch-injured BV2 transduced with empty vector lentivirus, $n=19$ ); the FPI+SImiR-5121 OE-B-EXO group (mice underwent FPI and received tail vein injection of exosomes derived from stretchinjured BV2 transduced with miR-5121 overexpression lentivirus, $n=19$ ); the FPI+SI-B-EXO+Saline group (mice underwent FPI and received tail vein injection of exosomes derived from stretch-injured BV2 and intraperitoneal injection of saline, $n=6$ ) and the FPI+SI-B-EXO+Y-27632 group (mice underwent FPI and received tail vein injection of exosomes derived from stretch-injured BV2 and intraperitoneal injection of Y-27632, $n=6$ ). All the animal experiments were approved by the Institutional Animal Care and Use Committee of Sun Yat-sen University and performed in accordance with the National Institute of Health Guide for the Care and Use of Laboratory Animals.

\section{Lateral Fluid Percussion Injury and Tail Vein Injections}

Lateral FPI was performed as previously described [17, 26]. Briefly, the mice were anesthetized with $4 \%$ isoflurane in an induction chamber and then maintained with $2 \%$ isoflurane via a nose cone after stabilizing in a stereotaxic frame (Stoelting, Varese, Italy). The body temperature of the mice was maintained at $37^{\circ} \mathrm{C}$ using a homoeothermic blanket. A 3$\mathrm{mm}$ diameter circular craniotomy was drilled on the left parietal bone $(0.5 \mathrm{~mm}$ lateral to the sagittal suture and midway between the bregma and lambda). Then the skullcap was removed, leaving the dura intact. Superglue and dental acrylic were used to secure the Luer Lock needle hub over the craniotomy hole. FPI was induced by the fluid percussion device (VCU Biomedical Engineering, Richmond, VA, USA). The peak impact pressure was controlled at 1.9-2.1 atm in order to induce moderate TBI.

Mice in the FPI+CON-B-EXO group, FPI+SI-B-EXO group, FPI+SI-NC-B-EXO group, FPI+SI-miR-5121 OE-BEXO group, FPI+SI-B-EXO+Saline group, and FPI+SI-B$\mathrm{EXO}+\mathrm{Y}-27632$ group were intravenously injected with microglia-derived exosomes (approximately $2.5 \times 10^{10}$ particles per mouse) by tail vein injection immediately after FPI, while mice in the FPI+PBS group was injected with the same volume of PBS. Mice in the FPI+SI-B-EXO+Y-27632 group were then intraperitoneally injected with Y-27632 (MedChemExpress, NJ, USA) at a concentration of $20 \mathrm{mg} / \mathrm{kg}$ body weight according to previous studies with Y27632 in animal disease models [27], while mice in the FPI+ SI-B-EXO+Saline group was injected with the same volume of saline. After $24 \mathrm{~h}$, all mice were sacrificed and the brains were collected for further experiments.

\section{Western Blot}

BV2 cells, exosomes, neurons, and pericontusional cortex were collected and lysed in RIPA buffer system (Santa Cruz, CA, USA). Protein concentrations were quantified with a BCA protein assay kit (Beyotime, Jiangsu, China). After being diluted in $5 \times$ SDS loading buffer and denatured at $100{ }^{\circ} \mathrm{C}$ for $5 \mathrm{~min}$, protein samples were separated by electrophoresis in $10 \%$ SDS-PAGE gel and transferred onto $0.22-\mu \mathrm{m}$ polyvinylidene difluoride membranes (Millipore, Merck KGaA, Darmstadt, Germany). The membranes were incubated in blocking buffer at room temperature for $1 \mathrm{~h}$ and then incubated with primary antibodies at $4{ }^{\circ} \mathrm{C}$ overnight. The following primary antibodies were used: mouse anti-Alix (1:1000; Cell Signaling); rabbit anti-CD63 (1:1000; Sigma); rabbit anti-CD81 (1:1000; Abcam); rabbit anti-growthassociated protein 43 (GAP43; 1:100,000; Abcam); rabbit 
anti-post-synaptic density-95 (PSD-95; 1:20,000; Abcam); rabbit anti-glutamate receptor 1 (GluR1; 1:5000; Abcam); rabbit anti-synaptophysin (1:100,000; Abcam); mouse antiglyceraldehyde 3-phosphate dehydrogenase (GAPDH; 1:10,000; Bioworld Technology); rabbit anti-repulsive guidance molecule family member A (RGMa; 1:25,000; Abcam); rabbit anti-ras homolog family member A (RhoA; 1:5000; Abcam); rabbit anti-Cleaved Caspase-3 (1:500; Cell Signaling); and rabbit anti-Cleaved PARP (1:1000, Cell Signaling). After being rinsed in TBST, the membranes were incubated at room temperature for $1 \mathrm{~h}$ in suitable secondary antibodies. The bands were detected using the ChemiDoc ${ }^{\mathrm{TM}}$ MP imaging system (Bio-Rad Laboratories, Cressier, Switzerland).

\section{Slice Preparation}

At 1 day after FPI, mice were transcardially perfused with $0.9 \%$ saline followed by $4 \%$ paraformaldehyde. After perfusion, the brains were removed, post-fixed in $4 \%$ phosphatebuffered paraformaldehyde at $4{ }^{\circ} \mathrm{C}$ overnight and then dehydrated in $15 \%, 20 \%$, and $30 \%$ gradient sucrose solutions for $48 \mathrm{~h}$. The brains were cut into $20-\mu \mathrm{m}$ coronal sections for immunofluorescence and $100-\mu \mathrm{m}$ coronal sections for dendrite spine quantification.

\section{Immunofluorescence}

The immunofluorescence was performed as previously described [28]. Briefly, the sections were rinsed in PBS and blocked in $10 \%$ goat serum containing $0.1 \%$ Triton X-100 for $1 \mathrm{~h}$. The neurons were rinsed in PBS and fixed in $4 \%$ PFA for $30 \mathrm{~min}$ at room temperature, followed by treating with $5 \%$ BSA containing $0.1 \%$ Triton $\mathrm{X}-100$ for $1 \mathrm{~h}$ at room temperature. The sections or neurons were then incubated with primary antibodies at $4{ }^{\circ} \mathrm{C}$ overnight. The following primary antibodies were used: mouse anti-MAP2 (1:200; Abcam); rabbit anti-GAP43 (1:200; Abcam); and mouse anti-NEUN (1:200; Abcam). After being rinsed in PBS, the sections or neurons were incubated with fluorescent-dye conjugated secondary antibodies at room temperature for $2 \mathrm{~h}$. The following secondary antibodies were used: goat anti-mouse IgG Alexa Fluor® 488 (1:500; Abcam) and goat anti-rabbit IgG Alexa Fluor® 594 (1:500; Abcam). The sections or neurons were then counterstained with DAPI and photographed by a Zeiss LSM 800 confocal microscope. The mean fluorescence intensity of PKH26 in the MAP2-labeled neurons in different groups was measured by ImageJ.

\section{Sholl Analysis}

Images of neurons were collected using a Zeiss LSM 800 confocal microscope and manually traced with simple neurite tracer software integrated in ImageJ. Dendritic tracings were then analyzed for total dendritic length and number of dendritic branching points. Dendritic complexity was further quantified by Sholl analysis. Sholl analysis was performed using regularly spaced concentric circles at $20-\mu \mathrm{m}$ distances. These circles were centered on the soma of a neuron. The number of dendrite intersections with each radius was quantified.

\section{Spine Quantification}

Spine quantification was performed as previously described [17]. The spines located at the main apical dendrite in layers $\mathrm{V} / \mathrm{VI}$ adjacent to the injury core were imaged for spine quantification by a Zeiss LSM 800 confocal microscope with $63 \times$ oil objective. Z-stacks were captured every $0.05 \mu \mathrm{m}$. Images were then reconstructed using a maximum intensity projection strategy. The total number of spines was quantified per $50-\mu \mathrm{m}$ length of dendrite using NeuronStudio.

\section{Beam Walking Test}

Beam walking test was performed to evaluate the motor coordination and balance of the mice [29]. The apparatus consisted of an uncovered starting stage, an enclosed goal box, and an $80-\mathrm{cm}$ beam with a flat surface of 3-, 2-, or 1-cm width. The mice were trained to traverse the beam placed $30 \mathrm{~cm}$ above the bench to reach the goal box. During training, the mice were placed on the starting stage and encouraged to cross the beam to escape an aversive stimulus near the starting stage. Each mouse learned to cross sequentially the $3-\mathrm{cm}$ beam, the $2-\mathrm{cm}$ beam, and the $1-\mathrm{cm}$ beam. The mice were trained for 2 days before surgery and tested at days 1 and 3 after TBI. The time required to cross the beam (latency) and the total number of times the hind feet slipped off the beam (foot-slips) were recorded for each mice.

\section{RNA Extraction and Quantitative Real-Time PCR}

MicroRNAs and mRNAs were extracted from microglial cells and microglia-derived exosomes using mirVana ${ }^{\mathrm{TM}}$ miRNA isolation kit (Ambion, Austin, TX, USA) according to manufacturer's instructions.

The mRNA was then reverse transcribed using transcriptor first-strand cDNA synthesis kit (Roche, Basel, Switzerland). Real-time PCR analysis was performed to quantify interleukin-1 $\beta$ (IL-1 $\beta$ ), interleukin-6 (IL-6), interleukin-10 (IL-10), inducible nitric oxide synthase (iNOS), Arginase, and CD206 mRNA expression levels using LightCycler® 480 SYBR® Green I Master (Roche, Basel, Switzerland). The primer sequences used were as follows: IL-1 $\beta, 5^{\prime}$-GAG CAC CTT CTT TTC CTT CAT CTT-3' (forward), 5'-TCA CAC ACC AGC AGG TTA TCA TC-3' (reverse); IL-6, 5'-CAA 
CGA TGA TGC ACT TGC AGA-3' (forward), 5'-CTC CAG GTA GCT ATG GTA CTC CAG A-3' (reverse); IL-10, 5'-GAT GCC CCA GGC AGA GAA-3' (forward), 5'-CAC CCA GGG AAT TCA AAT GC-3' (reverse); iNOS, 5'-TAG GCA GAG ATT GGA GGC CTT G-3' (forward), 5'-GGG TTG TTG CTG AAC TTC CAG TC-3' (reverse); Arginase, 5'-CAG AAG AAT GGA AGA GTC AG-3' (forward), 5'-CAG ATA TGC AGG GAG TCA CC-3' (reverse); CD206, 5'-CTT CGG GCC TTT GGA ATA AT-3' (forward), 5'-TAG AAG AGC CCT TGG GTT GA-3' (reverse); GAPDH, 5'-TGG GCT ACA CTG AGG ACC ACT-3' (forward), 5'-GGG AGT GTC TGT TGA AGT CG-3' (reverse). GAPDH was used as endogenous control for normalization of cellular mRNAs.

Exogenous Caenorhabditis elegans microRNA cel-miR39-3p (RiBoBio, Guangzhou, Guangdong, China) was added to each sample before isolation of exosomal microRNAs. cDNA synthesis and real-time PCR-based quantification of microRNA were performed using Applied Biosystems TaqMan ${ }^{\circ}$ microRNA assays. In brief, cDNA was synthesized using TaqMan® microRNA reverse transcription kit (Thermo Fisher Scientific, Waltham, MA, USA) following the manufacturer's protocol. Real-time PCR was carried out using TaqMan® universal master mix II, no AmpErase® UNG $(2 \times)$ (Thermo Fisher Scientific, Waltham, MA, USA) according to the manufacturer's instructions. Cel-miR-39-3p and U6 snRNA were used as exogenous control and endogenous control for normalization of exosomal and cellular microRNAs, respectively. Reverse transcription primers and real-time PCR primers of mmu-miR-5121 (Assay ID: 465019_mat), U6 (Assay ID: 001973) and cel-miR-39-3p (Assay ID: 000200) were purchased from Thermo Fisher Scientific (Waltham, MA, USA).

All reactions were run in triplicate and the data were analyzed using $2^{-\Delta \Delta \mathrm{Ct}}$ method.

\section{MicroRNA Microarray Analysis}

MicroRNA microarray analysis was performed by Shanghai Biotechnology Corporation. Total RNA was extracted from exosomes (exosomes derived from control BV2 and stretchinjured BV2) and then labeled with Cyanine3-pCp. Next, the labeled RNA was hybridized to Agilent Mouse miRNA $(8 * 60 \mathrm{~K})$ array (version 21.0$)$. Finally, microRNA microarrays were scanned and the data were extracted. MicroRNA with expression levels differing by at least threefold between exosomes derived from control BV2 and stretch-injured BV2 were selected.

\section{Lentiviral Vector Transduction}

BV2 cells were transduced with miR-5121 overexpression lentivirus (GeneChem, Shanghai, China) with multiplicity of infection of 100 , followed by incubation at $37^{\circ} \mathrm{C}$ in $5 \% \mathrm{CO}_{2}$ and replacement of fresh DMEM-High Glucose Medium with $10 \%$ FBS. The transduction efficiency was determined by detecting GFP-positive BV2 cells at 4 days after transduction. The empty vector lentivirus was used as a negative control.

\section{RhoA Activity Assay}

RhoA activity was measured by RhoA activation assay kit according to the manufacturer's instructions (NewEast Biosciences, PA, USA). One milligram of protein samples was incubated with $1 \mu \mathrm{L}$ anti-active RhoA monoclonal antibody and $20 \mu \mathrm{L}$ protein $\mathrm{A} / \mathrm{G}$ Agarose beads at $4{ }^{\circ} \mathrm{C}$ for $1 \mathrm{~h}$. The beads were then washed with wash buffer and resuspended in $20 \mu \mathrm{L}$ of $2 \times$ reducing SDSPAGE sample buffer. After denatured at $100{ }^{\circ} \mathrm{C}$ for $5 \mathrm{~min}$, the samples were then ready for western blot assay.

\section{Luciferase Reporter Assay}

Luciferase reporter assay was performed to verify whether miR-5121 targeted RGMa mRNA. In brief, the wild-type or mutant 3'UTR region of RGMa was inserted into the pmirGLO vector to generate wild-type or mutant RGMa 3' UTR luciferase vectors. 293T cells in a 96-well plate were transfected with these wild-type or mutant RGMa 3'UTR vectors. The cells were then co-transfected with miR-5121 mimic or miR-NC. At $48 \mathrm{~h}$ after transfection, luciferase activity was measured using the dual-luciferase reporter assay kit (Promega, Madison, WI, USA) in accordance with the manufacturer's instructions. Firefly luciferase activity was normalized to the corresponding Renilla luciferase activity.

\section{Statistical Analysis}

All data are presented as means \pm standard error of the mean. Statistical data analyses were performed using SPSS 16.0. Statistical comparisons between 2 groups were analyzed with Student's $t$ test. Statistical comparisons among 3 groups were analyzed with one-way analysis of variance (ANOVA) followed by Tukey's post hoc test. Statistical analysis of the beam walking test was performed using two-way repeated measures ANOVA with Bonferroni post hoc test. Differences in which $p<0.05$ were considered significant.

\section{Results}

\section{Severe Stretch Injury Induced Cell Death of Microglia and Significantly Increased the Expression Level of IL- $1 \beta$ mRNA in Microglia}

At $24 \mathrm{~h}$ after stretch-induced injury, cell viability of BV2 microglia was detected by CCK-8 assay. As shown in Fig. S1A, cell viability of BV2 microglia was significantly 
decreased after severe stretch-induced injury. Moreover, the expression levels of apoptotic marker proteins, Cleaved Caspase-3 and Cleaved PARP, in BV2 microglia were detected. Western blot analysis showed that the expression levels of Cleaved Caspase-3 and Cleaved PARP in BV2 microglia were significantly increased after severe stretch-induced injury (Fig. S1B).

Furthermore, the expression levels of IL-1 $\beta$, IL-6, IL-10, iNOS, Arginase, and CD206 mRNA in microglia at $24 \mathrm{~h}$ after stretch-induced injury were detected by real-time PCR. As shown in Fig. 1(A) and Fig. 1(B), the expression level of IL$1 \beta$ (M1 microglial marker) mRNA in BV2 microglia and primary microglia was significantly increased after severe

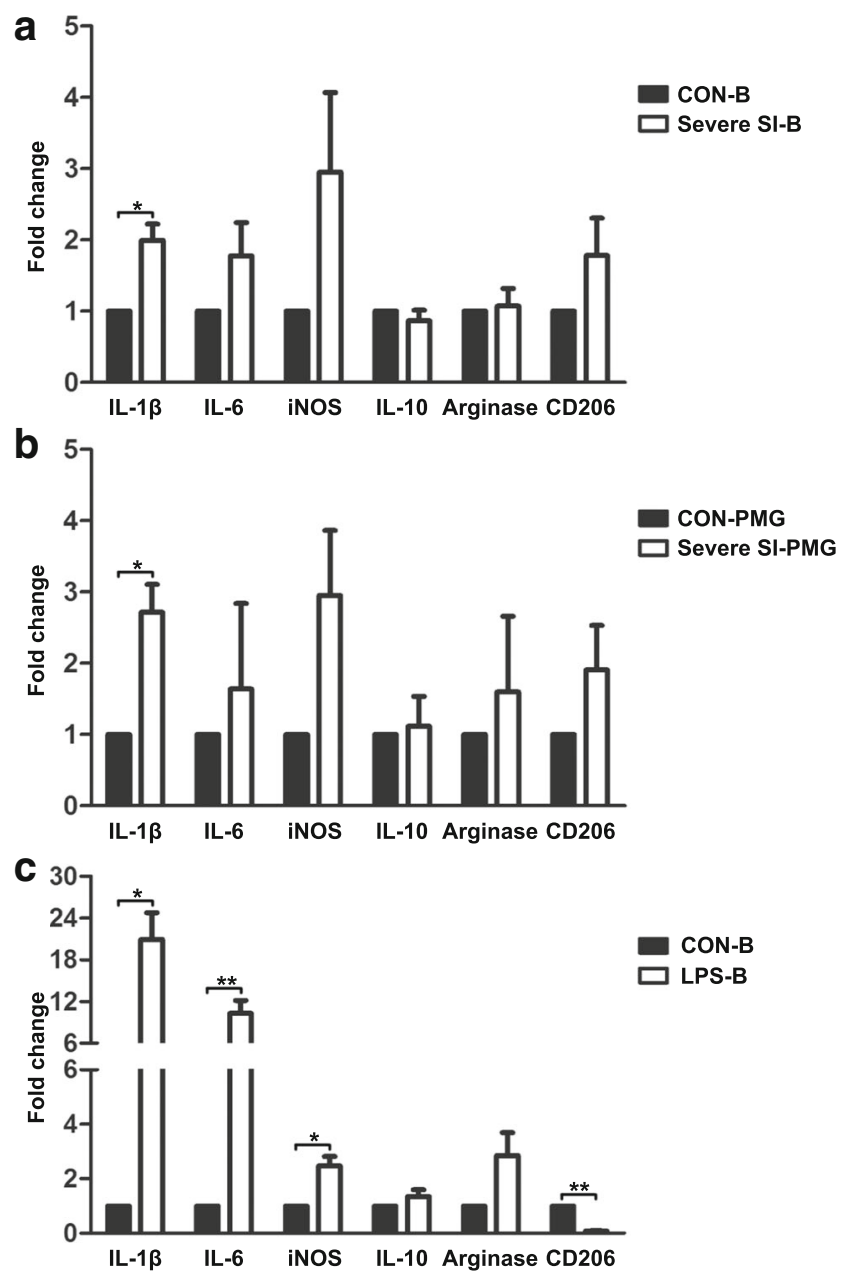

Fig. 1 (A) IL-1 $\beta$, IL-6, iNOS, IL-10, Arginase, and CD206 mRNA expression of BV2 microglia at $24 \mathrm{~h}$ after severe stretch-induced injury. (B) IL-1 $\beta$, IL-6, iNOS, IL-10, Arginase, and CD206 mRNA expression of primary microglia at $24 \mathrm{~h}$ after severe stretch-induced injury. (C) IL$1 \beta$, IL-6, iNOS, IL-10, Arginase, and CD206 mRNA expression of BV2 microglia at $24 \mathrm{~h}$ after LPS stimulation. Quantification revealed that the expression level of IL-1 $\beta$ mRNA was significantly increased in BV2 microglia and primary microglia after severe stretch-induced injury. The expression levels of IL-1 $\beta$, IL-6, and iNOS mRNA were significantly increased and the expression level of CD206 was significantly decreased in BV2 microglia at $24 \mathrm{~h}$ after LPS stimulation. All data are represented as the mean $\pm \mathrm{SEM} ; n=3$; two-tailed $t$ tests was used to analyze significant differences. $* p<0.05, * * p<0.01$ stretch-induced injury. However, the expression levels of IL6, iNOS (M1 microglial markers), IL-10, Arginase, and CD206 (M2 microglial markers) mRNA in BV2 microglia and primary microglia showed no significant difference after severe stretch-induced injury. Moreover, the expression levels of IL-1 $\beta$, IL-6, iNOS, IL-10, Arginase, and CD206 mRNA in BV2 microglia did not significantly change at $24 \mathrm{~h}$ after mild stretch-induced injury (Fig. S2A).

We also measured the expression levels of IL- $1 \beta$, IL-6, IL10, iNOS, Arginase, and CD206 mRNA in BV2 microglia at $24 \mathrm{~h}$ after LPS stimulation. As shown in Fig. 1(C), the expression levels of IL-1 $\beta$, IL- 6 , and iNOS mRNA were significantly increased and the expression level of CD206 was significantly decreased after LPS stimulation.

These results demonstrated that microglia were activated and polarized to pro-inflammation (M1) phenotype at $24 \mathrm{~h}$ after severe stretch-induced injury.

\section{Secretion of Exosomes from Microglia Was Significantly Increased After Severe Stretch-Induced Injury and LPS Stimulation}

Exosomes secreted from uninjured microglia, stretch-injured microglia, and LPS-stimulated microglia were isolated using ultracentrifugation and identified by TEM, western blot, and NTA. As shown in Fig. 2(A), the particles isolated from uninjured BV2 microglia and stretch-injured BV2 microglia had a typical cupshaped morphology and a diameter in the range of 40-150 nm. Western blot analysis showed that characteristic exosome biomarkers, including Alix, CD63, and CD81, were expressed in the particles isolated from uninjured BV2 microglia and stretchinjured BV2 microglia (Fig. 2(B)). Furthermore, the concentration and size distribution of the isolated particles were analyzed by NTA. As shown in Fig. 2(C, D) and S2B, the peak diameters of particles isolated from uninjured BV2 microglia, mild stretchinjured BV2 microglia, severe stretch-injured BV2 microglia, and LPS-stimulated BV2 microglia were both around $100 \mathrm{~nm}$. The total concentration of exosomes isolated from BV2 microglia was significantly increased after severe stretch-induced injury and LPS stimulation, but did not change after mild stretchinduced injury. Moreover, the total concentration of exosomes isolated from primary microglia was also significantly increased after severe stretch-induced injury (Fig. 2(E)).

These results revealed that secretion of exosomes from microglia was significantly increased after severe stretchinduced injury.

\section{Exosomes Derived from Stretch-Injured BV2 Microglia Suppressed Neurite Outgrowth and Synapse Recovery In Vitro}

To investigate the influence of microglia-derived exosomes on neurite outgrowth and synapse recovery after TBI 
Fig. 2 Characterization of exosomes derived from microglia. (A) The structure of uninjured BV2 and stretchinjured BV2-derived exosomes observed by TEM. (B) Western blot analysis of exosomal surface markers Alix, CD63, and CD81 in BV2-derived exosomes. (C) Particle size distribution and total concentration of uninjured BV2 and stretch-injured BV2-derived exosomes measured by nanoparticle tracking analysis. (D)

Particle size distribution and total concentration of LPS-stimulated

BV2-derived exosomes measured by nanoparticle tracking analysis. (E) Particle size distribution and total concentration of uninjured primary microglia and stretchinjured primary microglia-derived exosomes measured by nanoparticle tracking analysis.

Quantification revealed that the total concentration of microgliaderived exosomes was significantly increased in the Severe SIB-EXO group and LPS-B-EXO group compared to that of the CON-B-EXO group. The total concentration of microgliaderived exosomes was significantly increased in the Severe SIPMG-EXO group compared to that of the CON-PMG-EXO group. All data are represented as the mean \pm SEM; $n=3$; twotailed $t$ tests was used to analyze significant differences. ${ }^{*} p<0.05$, $* * p<0.01$
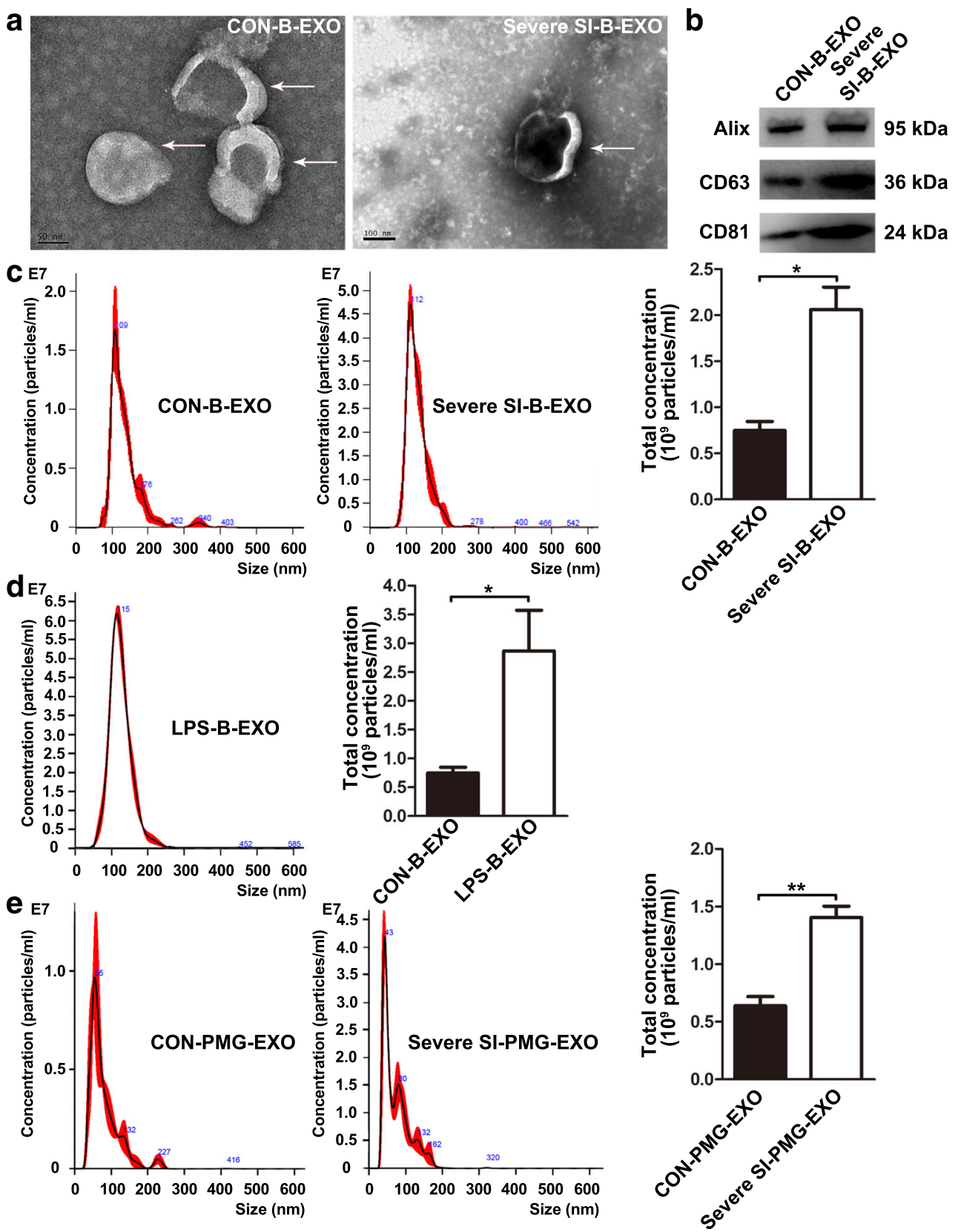

in vitro, exosomes isolated from uninjured microglia and stretch-injured BV2 microglia (approximately $2.5 \times 10^{8}$ particles $/ \mathrm{ml}$ ) were labeled with PKH26 (red fluorescence) and cocultured with stretch-injured neurons for $24 \mathrm{~h}$ (Fig. 3(A)). After 24-h co-culture, immunofluorescence staining was performed to verify if microglia-derived exosomes could be taken up by neurons in vitro. As shown in Fig. 3(B), PKH26-labeled exosomes were localized in the cytoplasm of MAP2positive neurons, suggesting neurons could take up microglia-derived exosomes in vitro. In addition, after 24-h co-culture with PKH26-labeled exosomes, the mean fluorescence intensity of neurons was measured to evaluate the amount of exosomes taken up by neurons. Flow cytometry analysis and immunofluorescence showed an increase in the mean fluorescence intensity of PKH26 in stretch-injured neurons compared with uninjured neurons after addition of PKH26-labeled CON-B-EXO or SI-BEXO. Moreover, the uninjured neurons or stretch-injured neurons co-cultured with PKH26-labeled CON-B-EXO had a higher mean fluorescence intensity of PKH26 compared with that co-cultured with SI-B-EXO (Fig. S3A, B, $\mathrm{C}$, and $\mathrm{D})$. These results revealed that stretch-injured neurons took up more exosomes than uninjured neurons, and neurons took up more exosomes derived from uninjured BV2 microglia than that derived from stretch-injured BV2 microglia. 


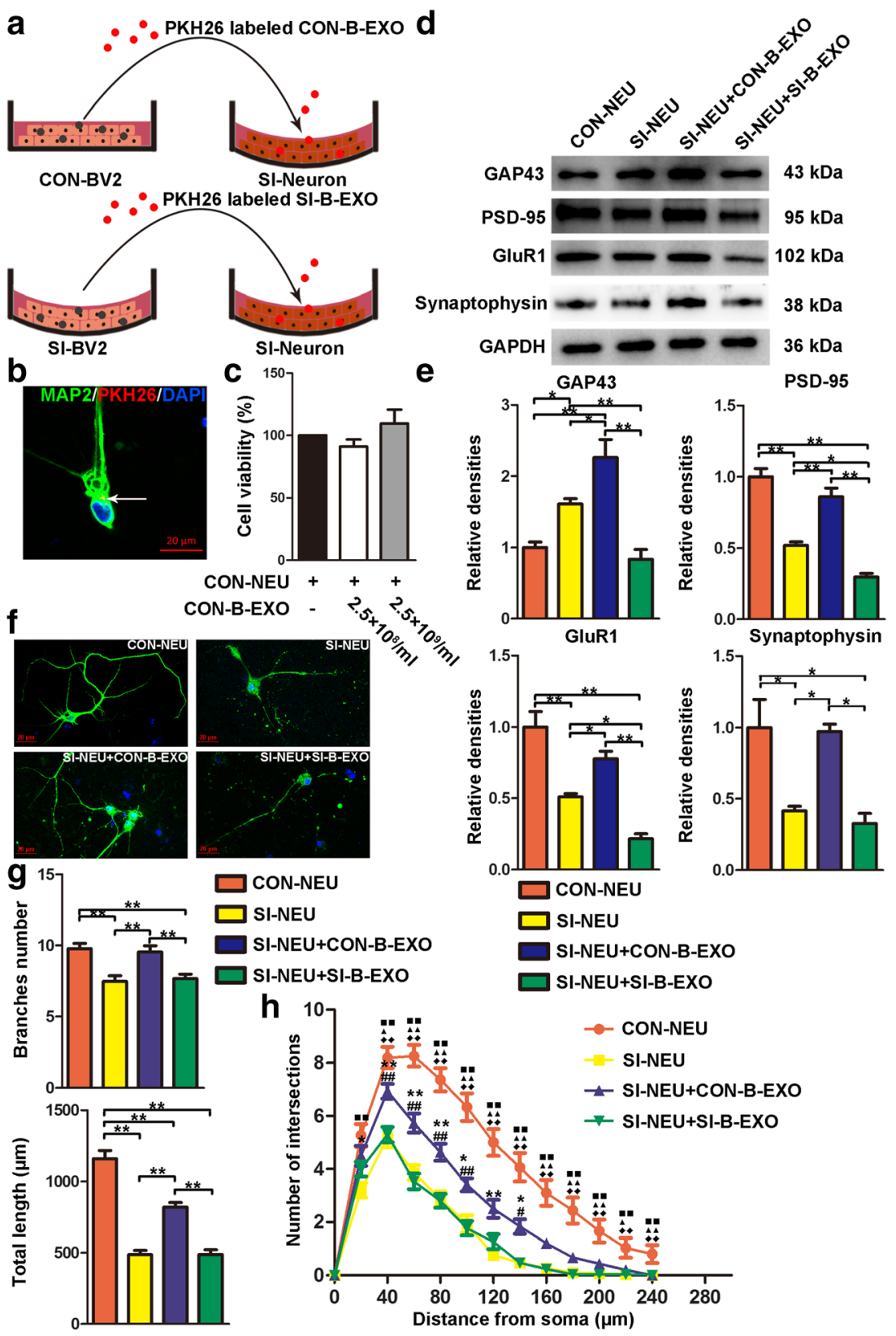

Fig. 3 Exosomes derived from stretch-injured BV2 microglia suppressed neurite outgrowth and synapse recovery in vitro. (A) A schematic diagram illustrating fluorescent labeling of uninjured BV2 and stretchinjured BV2-derived exosomes with PKH26 and co-culture of PKH26labeled exosomes with stretch-injured neurons. (B) Confocal imaging showed that PKH26 (red)-labeled exosomes derived from BV2 microglia were taken up by neurons in vitro. Neurons were identified by MAP2 (green). (C) Cell viability of neurons co-cultured with two concentrations of uninjured BV2-derived exosomes $\left(2.5 \times 10^{8} / \mathrm{ml}\right.$ and $\left.2.5 \times 10^{9} / \mathrm{ml}\right)$ was detected by CCK-8 assay. Quantification revealed that uninjured BV2derived exosomes $\left(2.5 \times 10^{8} / \mathrm{ml}\right.$ and $\left.2.5 \times 10^{9} / \mathrm{ml}\right)$ showed no significant cytotoxicity against neurons after $24 \mathrm{~h}(n=3)$. (D) Representative immunoblots of GAP43, PSD-95, GluR1, and Synaptophysin in the CONNEU group, SI-NEU group, SI-NEU+CON-B-EXO group, and SINEU+SI-B-EXO group. (E) Quantification revealed that the protein levels of GAP43, PSD-95, GluR1, and Synaptophysin were decreased in stretch-injured neurons co-cultured with exosomes derived from stretch-injured BV2 microglia compared with that of exosomes derived from uninjured BV2 microglia. (F) Representative confocal imaging of uninjured neurons, stretch-injured neurons, and stretch-injured neurons co-cultured with uninjured BV2 and stretch-injured BV2-derived exosomes. Neurons were identified by MAP2 (green). (G) Quantified results of neurite analysis revealed that branch number and total length were significantly decreased in stretch-injured neurons co-cultured with exosomes derived from stretch-injured BV2 microglia compared with that of exosomes derived from uninjured BV2 microglia. (H) Sholl analysis identified a decrease in the number of intersections 40, 60, 80, 100, and $140 \mu \mathrm{m}$ from the neuron soma in the SI-NEU+SI-B-EXO group compared with that of the SI-NEU+CON-B-EXO group. Two black squares, $p<0.01$, CON-NEU versus SI-NEU; one black triangle, $p<0.05$, two black triangles, $p<0.01$, CON-NEU versus SI-NEU+ CON-B-EXO; two black diamonds, $p<0.01$, CON-NEU versus SINEU+SI-B-EXO; $* p<0.05, * * p<0.01$, SI-NEU versus SI-NEU+ CON-B-EXO; $\# p<0.05, \# \# p<0.01$, SI-NEU+CON-B-EXO versus SINEU+SI-B-EXO. All data are represented as the mean \pm SEM; $n=6$; one-way ANOVA with Tukey's post hoc test was used to analyze significant differences. $* p<0.05$, $* p<0.01$ 
To measure the side effects of microglia-derived exosomes, neurons were co-cultured with different doses of microgliaderived exosomes $\left(2.5 \times 10^{8}\right.$ particles $/ \mathrm{ml}$ and $2.5 \times 10^{9}$ particles $/ \mathrm{ml}$ ) for $24 \mathrm{~h}$. A CCK-8 assay was then performed to evaluate cell viability. The result revealed no significant cytotoxicity of microglia-derived exosomes $\left(2.5 \times 10^{8}\right.$ particles $/ \mathrm{ml}$ and $2.5 \times 10^{9}$ particles $/ \mathrm{ml}$ ) against neurons after co-cultured for $24 \mathrm{~h}$ (Fig. 3(C)).

We next detected the expression levels of GAP43, PSD-95, GluR1, and Synaptophysin in stretch-injured neurons after coculture with exosomes derived from uninjured BV2 microglia or stretch-injured BV2 microglia. As shown in Fig. 3(D) and Fig. 3(E), the protein levels of GAP43, PSD-95, and GluR1 were significantly decreased in stretch-injured neurons cocultured with exosomes derived from stretch-injured BV2 microglia compared with that of the SI-NEU group and SI$\mathrm{NEU}+\mathrm{CON}-\mathrm{B}-\mathrm{EXO}$ group. The protein level of Synaptophysin in the SI-NEU+SI-B-EXO group showed no significant difference compared with that of the SI-NEU group, but was significantly lower than that of the SI-NEU+ CON-B-EXO group. Moreover, co-cultured with exosomes derived from uninjured BV2 microglia could significantly increase the protein levels of GAP43, PSD-95, GluR1, and Synaptophysin in stretch-injured neurons. The protein levels of PSD-95, GluR1, and Synaptophysin showed no significant difference between the CON-NEU group and SI-NEU+CONB-EXO group.

To further determine the effects of microglia-derived exosomes on neuronal dendrite structure after stretchinduced injury, neurons were stained with MAP2 (Fig. 3(F)) and the morphology changes were assessed. As shown in Fig. $3(\mathrm{G})$, the number of dendrite branches and their total length were significantly decreased in the SI-NEU+SI-B-EXO group compared with the SI-NEU+CON-B-EXO group and CONNEU group, but showed no significant difference between the SI-NEU group and SI-NEU+SI-B-EXO group. Moreover, cocultured with exosomes derived from uninjured BV2 microglia could significantly increase the branches number and total length of the neurons after stretch-induced injury. The number of dendrite branches showed no significant difference between the CON-NEU group and SI-NEU+CON-B-EXO group. In addition, a Sholl analysis of the neurons was performed to evaluate the changes in dendrite complexity. As shown in Fig. 3(H), the dendrites of SI-B-EXO-treated neurons showed less intersections than those of CON-B-EXOtreated neurons at $40,60,80,100$, and $140 \mu \mathrm{m}$ from the neuron soma and those of uninjured neurons between 40 and $240 \mu \mathrm{m}$ from the neuron soma. However, the dendrite complexity showed no significant difference between the SI-NEU group and SI-NEU+SI-B-EXO group. Moreover, the dendrites of CON-B-EXO-treated neurons showed more intersections than those of stretch-injured neurons between 20 and $140 \mu \mathrm{m}$ from the neuron soma and less intersections than those of uninjured neurons between 40 and $240 \mu \mathrm{m}$ from the neuron soma.

These results revealed that microglia-derived exosomes could be taken up by neurons and exosomes derived from stretch-injured BV2 microglia suppressed neurite outgrowth and synapse recovery in vitro.

\section{Exosomes Derived from Stretch-Injured BV2 Microglia Suppressed Neurite Outgrowth and Synapse Recovery In Vivo}

To determine whether exosomes derived from stretch-injured microglia also suppressed neurite outgrowth and synapse recovery in vivo, Thy1-GFP knock-in mice were intravenously injected with PKH26-labeled exosomes isolated from uninjured BV2 microglia or stretch-injured BV2 microglia through tail vein injection following FPI (Fig. 4(A)). Immunofluorescence staining was performed to verify if microglia-derived exosomes could penetrate blood-brain barrier and be taken up by neurons in vivo. As shown in Fig. 4(B) and Fig. 4(C), PKH26-labeled exosomes were detected in layer V/VI GFP+ pyramidal neurons and GFP-/MAP2+ neurons in other layers.

We next performed dual-immunofluorescence staining of GAP43 and NEUN, western blot analysis of GAP43, and spine quantification in the pericontusion region to evaluate the influence of microglia-derived exosomes on neurite outgrowth and synapse recovery following FPI. As shown in Fig. 4(E), GAP43+ NEUN cell percentage in the pericontusion region (within $1 \mathrm{~mm}$ from the edge of injury core, Fig. 4(D)) was significantly decreased in the FPI+SI-B-EXO group compared with that of the FPI+PBS group and FPI+CON-B-EXO group 1 day following FPI. Moreover, tail vein injection of exosomes isolated from uninjured BV2 microglia could significantly increase GAP43+ NEUN cell percentage in the pericontusion region following FPI. In addition, we also detected the expression level of GAP43 in the pericontusion region by western blot. As shown in Fig. 4(F), the expression level of GAP43 was significantly decreased in the FPI+SI-BEXO group compared with that of the FPI+PBS group and FPI+CON-B-EXO group. Moreover, the expression level of GAP43 was significantly increased in the FPI+CON-B-EXO group compared with that of the FPI+PBS group.

Furthermore, spine quantification showed that apical dendritic spine density in the pericontusion region was also significantly decreased in the FPI+SI-B-EXO group compared with that of the FPI+PBS group and FPI+CON-B-EXO group 1 day following FPI (Fig. 4(G)). Tail vein injection of exosomes isolated from uninjured BV2 microglia could significantly increase apical dendritic spine density in the pericontusion region. However, the apical dendritic spine density in the FPI+CON-B-EXO group still did not reach the normal level. 

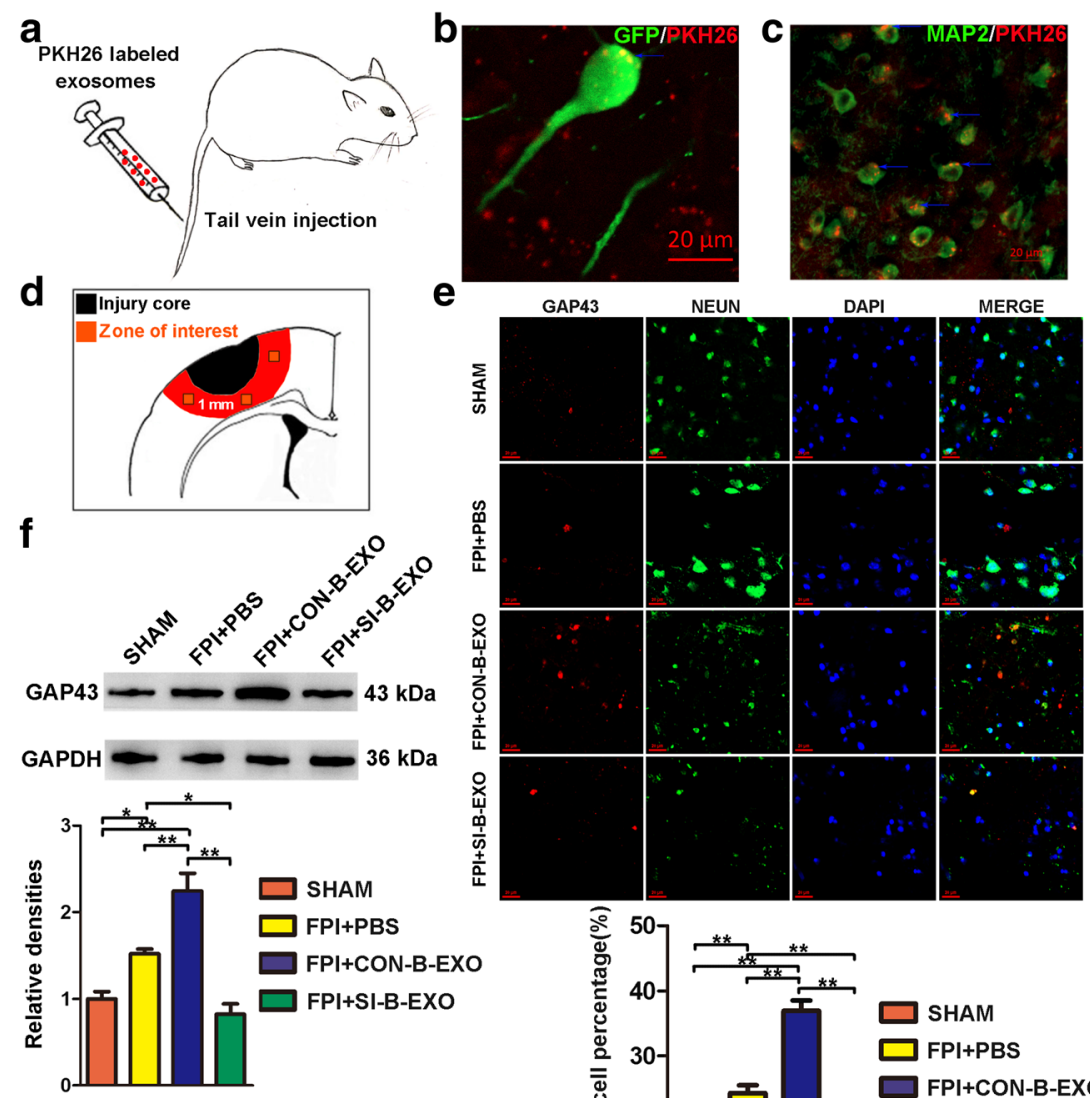

g
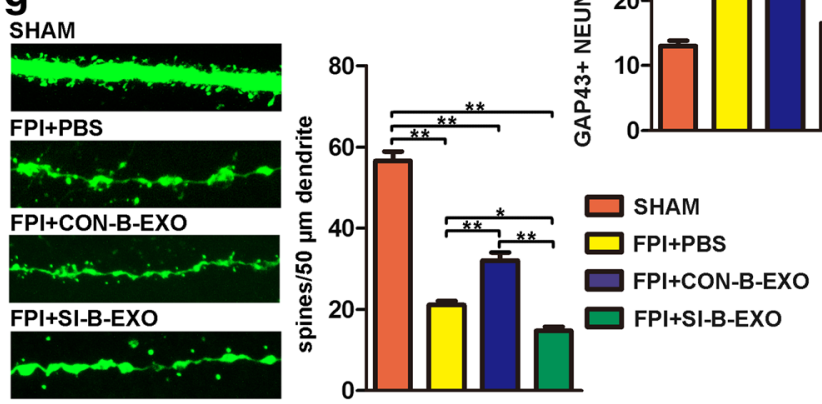

Fig. 4 Tail vein injection of exosomes derived from stretch-injured BV2 microglia suppressed neurite outgrowth and synapse recovery in vivo. (A) A schematic diagram illustrating tail vein injection of PKH26labeled exosomes. (B) Representative confocal imaging of PKH26labeled exosomes absorbed by GFP+ pyramid neurons in the pericontusion region. (C) Representative confocal imaging of PKH26labeled exosomes absorbed by GFP- neurons in the pericontusion region. Neurons were identified by MAP2 (green). (D) A schematic diagram of regions of interest (within $1 \mathrm{~mm}$ from the edge of injury core). Images were taken at both sides and the bottom of regions of interest (squares). (E) Detection of GAP43 expression in neurons by immunofluorescence. Quantification revealed that tail vein injection of exosomes derived from stretch-injured BV2 microglia decreased the numbers of GAP43+

These results revealed that microglia-derived exosomes could penetrate blood-brain barrier and be taken up by neurons, and exosomes derived from stretch-injured BV2 microglia suppressed neurite outgrowth and synapse recovery in vivo. neurons. Neurons were identified by NEUN (green). (F) Representative immunoblots of GAP43 in the SHAM group, FPI+PBS group, FPI+ CON-B-EXO group, and FPI+SI-B-EXO group. Quantification revealed that tail vein injection of exosomes derived from stretch-injured BV2 microglia significantly decreased the protein level of GAP43 in the pericontusion region. (G) Representative confocal imaging of apical dendritic spines in the pericontusion region (layers V/VI). Quantification revealed that tail vein injection of exosomes derived from stretchinjured BV2 microglia decreased apical dendritic spine density in the pericontusion region after FPI. All data are represented as the mean \pm SEM; $n=6$; one-way ANOVA with Tukey's post hoc test was used to analyze significant differences. ${ }^{*} p<0.05$, ${ }^{* *} p<0.01$

\section{Exosomes Derived from Stretch-Injured BV2 Microglia Impaired Motor Coordination in Mice after FPI}

To determine whether exosomes derived from stretch-injured BV2 microglia impaired neurological functions, the beam 
walking test was performed to evaluate the motor coordination and balance of the mice. At 1 day after FPI, SI-B-EXO-treated mice performed a significant increase in time and percentage of foot-slips when they crossed the 2-cm and 1-cm width beams in comparison to CON-B-EXO-treated mice (Fig. 5(A, B)). However, for the 3-cm width beam, SI-BEXO-treated mice only performed a significant increase in time to cross the beam and showed no significant difference in percentage of foot-slips compared with CON-B-EXOtreated mice.
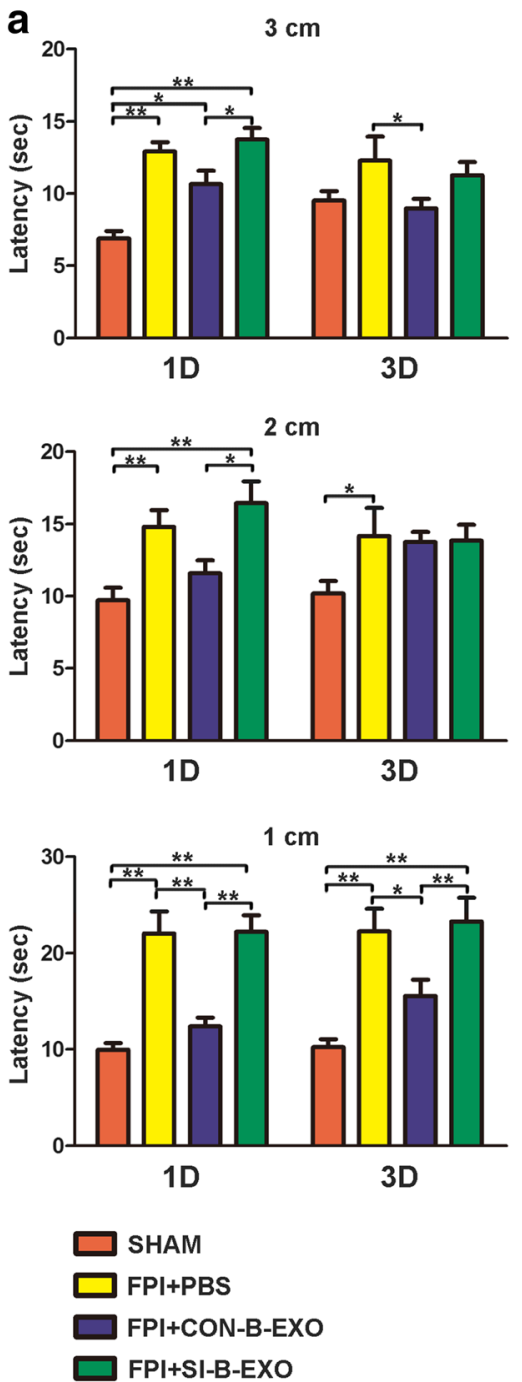

Fig. 5 Tail vein injection of exosomes derived from stretch-injured BV2 microglia impaired motor coordination in mice after FPI. (A) Latency and (B) percentage of foot-slips in the beam walking test at 1 and 3 days following FPI were measured. Quantification revealed that SI-B-EXOtreated mice performed a significant increase in time and percentage of foot-slips when they crossed the 2-cm and 1-cm width beams in comparison to CON-B-EXO-treated mice at 1 day after FPI. For the $3-\mathrm{cm}$ width beam, SI-B-EXO-treated mice only performed a significant increase in time to cross the beam and showed no significant difference in percentage of foot-slips compared with CON-B-EXO-treated mice. At 3 days after FPI, SI-B-EXO-treated mice performed a significant increase in time and
At 3 days after FPI, SI-B-EXO-treated mice performed a significant increase in time and percentage of foot-slips when they crossed the 1-cm width beam in comparison to CON-BEXO-treated mice (Fig. 5(A, B)). There was no significant difference between SI-B-EXO-treated and CON-B-EXOtreated mice in time and percentage of foot-slips to cross the $3-\mathrm{cm}$ and 2-cm width beams.

Moreover, there was no significant difference between SIB-EXO-treated mice and PBS-treated mice in time and
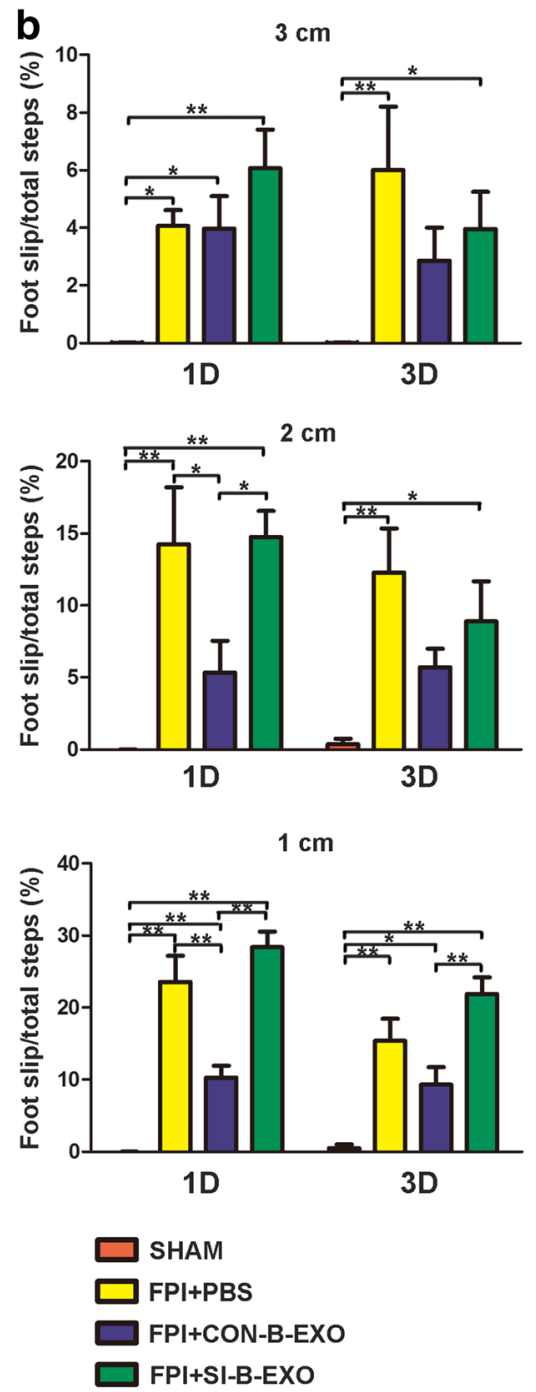

percentage of foot-slips when they crossed the 1-cm width beam in comparison to CON-B-EXO-treated mice. There was no significant difference between SI-B-EXO-treated and CON-B-EXO-treated mice in time and percentage of foot-slips to cross the $3-\mathrm{cm}$ and $2-\mathrm{cm}$ width beams. Moreover, mice in the FPI+SI-B-EXO group showed no significant difference compared to mice in the FPI+PBS group in latency and percentage of foot-slips at both 1 and 3 days following FPI. All data are represented as the mean $\pm \mathrm{SEM} ; n=7$; two-way repeated measures ANOVA with Bonferroni post hoc test was used to analyze significant differences. $* p<0.05, * * p<0.01$ 


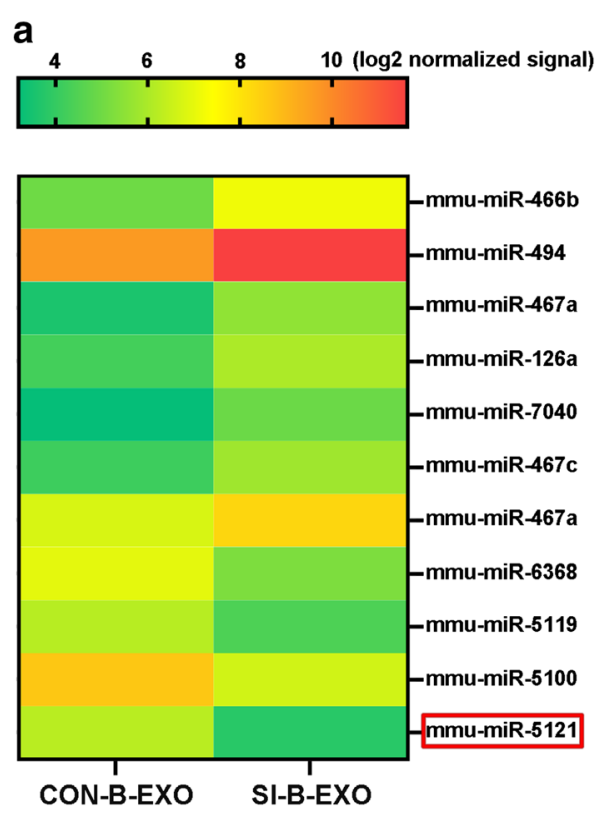

Fig. 6 The level of miR-5121 was decreased in exosomes derived from stretch-injured microglia and LPS-stimulated microglia. (A) Heat map of microRNA expression normalized data of exosomes derived from control and injured BV2 microglia detected by microarray analysis. MicroRNAs with fold change $>3$ were displayed in heat map. (B) The miR-5121 level in exosomes derived from microglia was detected by quantitative real-

percentage of foot-slips to cross the 3-cm, 2-cm, and $1-\mathrm{cm}$ width beams at both 1 and 3 days after FPI.

These results revealed that exosomes derived from stretchinjured BV2 microglia impaired neurological functions of the mice after FPI.

\section{Elevated Exosomal miR-5121 Promoted Neurite Outgrowth and Synapse Recovery both In Vitro and In Vivo}

To investigate the expression profile change of exosomal microRNAs derived from microglia after stretch-induced injury, we performed microRNA microarray analysis. As shown in Fig. 6(A) and Table S1, the expression level of exosomal miR-5121 was decreased most greatly after stretch-induced injury of BV2 microglia. Further quantitative real-time PCR verified that the expression level of miR-5121 was decreased in exosomes derived from stretch-injured BV2 microglia and LPS-stimulated BV2 microglia compared with that of uninjured BV2 microglia. The expression level of miR-5121 was also decreased in stretch-injured primary microglia-derived exosomes compared with uninjured primary microgliaderived exosomes (Fig. 6(B)).

To further investigate whether exosomal miR-5121 was involved in neurite outgrowth and synapse recovery, BV2 microglia were transduced with miR-5121 overexpression

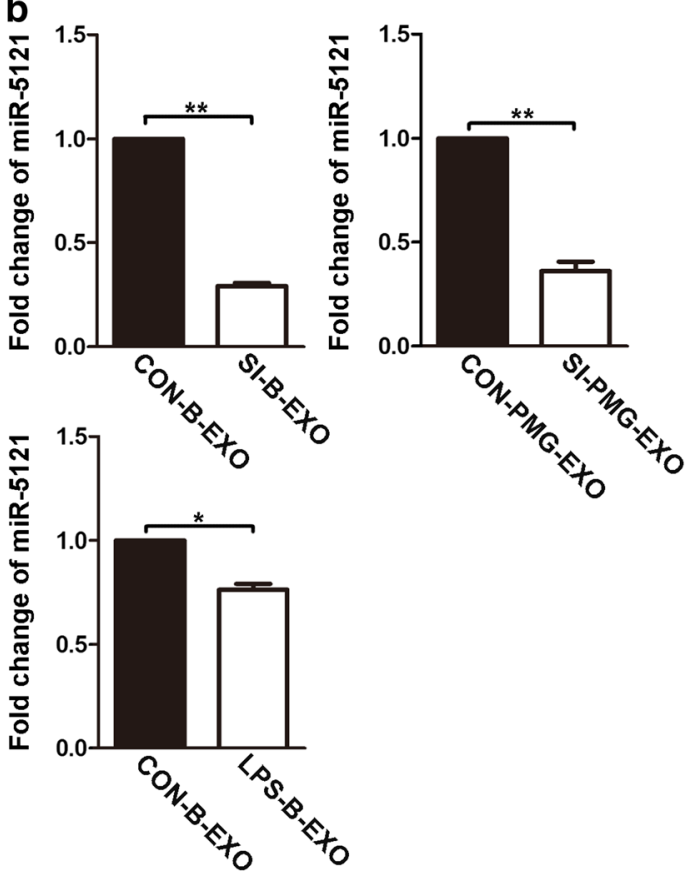

time PCR. Quantification revealed that the level of miR-5121 were decreased in exosomes derived from stretch-injured BV2 microglia, stretchinjured primary microglia, and LPS-stimulated microglia. All data are represented as the mean $\pm \mathrm{SEM} ; n=3$; two-tailed $t$ tests was used to analyze significant differences. $* p<0.05, * * p<0.01$

lentivirus and their derived exosomes were co-cultured with stretch-injured neurons in vitro. Quantitative real-time PCR verified that the expression level of miR-5121 was increased in both BV2 microglia and their derived exosomes after transducing with miR-5121 overexpression lentivirus (Fig. 7(A)). Western blot analysis showed that the protein levels of GAP43, PSD-95, GluR1, and Synaptophysin in stretchinjured neurons were significantly increased in the SI-NEU+ SI-miR-5121 OE-B-EXO compared with that of the SI-NEU+ SI-B-EXO group and SI-NEU+SI-NC-B-EXO group (Fig. 7(B) and Fig. 7(C)). Moreover, the protein levels of PSD-95, GluR1, and Synaptophysin in stretch-injured neurons showed no significant difference between the CON-NEU group and SI-NEU+SI-miR-5121 OE-B-EXO group.

To further determine the effects of exosomal miR-5121 derived from microglia on neuronal dendrite structure after stretch-induced injury, neurons were stained with MAP2 (Fig. 7(D)) and the morphology changes were assessed. As shown in Fig. 7(E), the total length of dendrite branches was significantly increased in the SI-NEU+SI-miR-5121 OE-BEXO group compared with that of the SI-NEU+SI-B-EXO group and SI-NEU+SI-NC-B-EXO group. However, the number of dendrite branches showed no significant difference among the SI-NEU+SI-B-EXO group, SI-NEU+SI-NC-BEXO group, and SI-NEU+SI-miR-5121 OE-B-EXO group. The number and total length of dendrite branches were all 

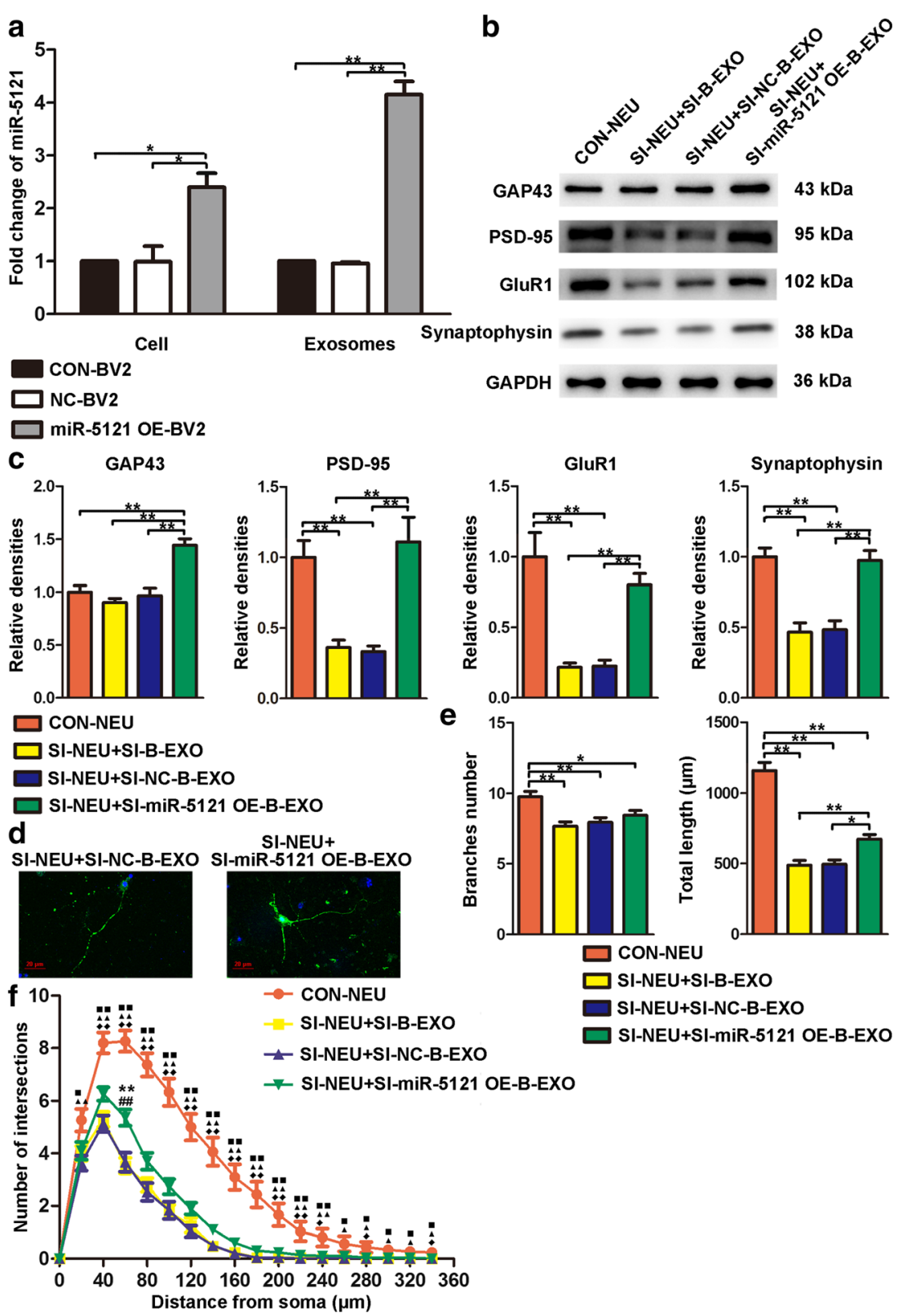

Fig. 7 MiR-5121 overexpressed exosomes derived from stretch-injured BV2 microglia promoted neurite outgrowth and synapse recovery in vitro. (A) The miR-5121 levels in BV2 microglia and exosomes derived from BV2 microglia were detected by quantitative real-time PCR. Quantification revealed that the levels of miR-5121 were elevated both in $\mathrm{BV} 2$ microglia and in exosomes derived from BV2 microglia in the miR5121 OE-BV2 group $(n=3)$. (B) Representative immunoblots of GAP43, PSD-95, GluR1, and Synaptophysin in the CON-NEU group, SI-NEU+ SI-B-EXO group, SI-NEU+SI-NC-B-EXO group, and SI-NEU+SI-miR5121 OE-B-EXO group. (C) Quantification revealed that the protein levels of GAP43, PSD-95, GluR1, and Synaptophysin were significantly increased in stretch-injured neurons co-cultured with miR-5121 overexpressed exosomes derived from stretch-injured BV2 microglia. (D) Representative confocal imaging of stretch-injured neurons cocultured with exosomes derived from stretch-injured negative control BV2 and stretch-injured miR-5121 overexpressed BV2. Neurons were identified by MAP2 (green). (E) Quantified results of neurite analysis revealed that total length was significantly increased in stretch-injured neurons co-cultured with miR-5121 overexpressed exosomes derived from stretch-injured BV2 microglia. (F) Sholl analysis identified an increase in the number of intersections $60 \mu \mathrm{m}$ from the neuron soma in the SI-NEU+SI-miR-5121 OE-B-EXO group compared with that of the SI$\mathrm{NEU}+\mathrm{SI}-\mathrm{B}-\mathrm{EXO}$ group and SI-NEU+SI-NC-B-EXO group. One black square, $p<0.05$; two black squares, $p<0.01$, CON-NEU versus SINEU+SI-B-EXO; one black triangle, $p<0.05$; two black triangles, $p<0.01$, CON-NEU versus SI-NEU+SI-NC-B-EXO; one black diamond, $p<0.05$; two black diamonds, $p<0.01$, CON-NEU versus SINEU+SI-miR-5121 OE-B-EXO; $* * p<0.01$, SI-NEU+SI-B-EXO versus SI-NEU+SI-miR-5121 OE-B-EXO; \#\# $p<0.01$, SI-NEU+SI-NC-BEXO versus SI-NEU+SI-miR-5121 OE-B-EXO. All data are represented as the mean $\pm \mathrm{SEM} ; n=6$; one-way ANOVA with Tukey's post hoc test was used to analyze significant differences. ${ }^{*} p<0.05$, * $p<0.01$ 

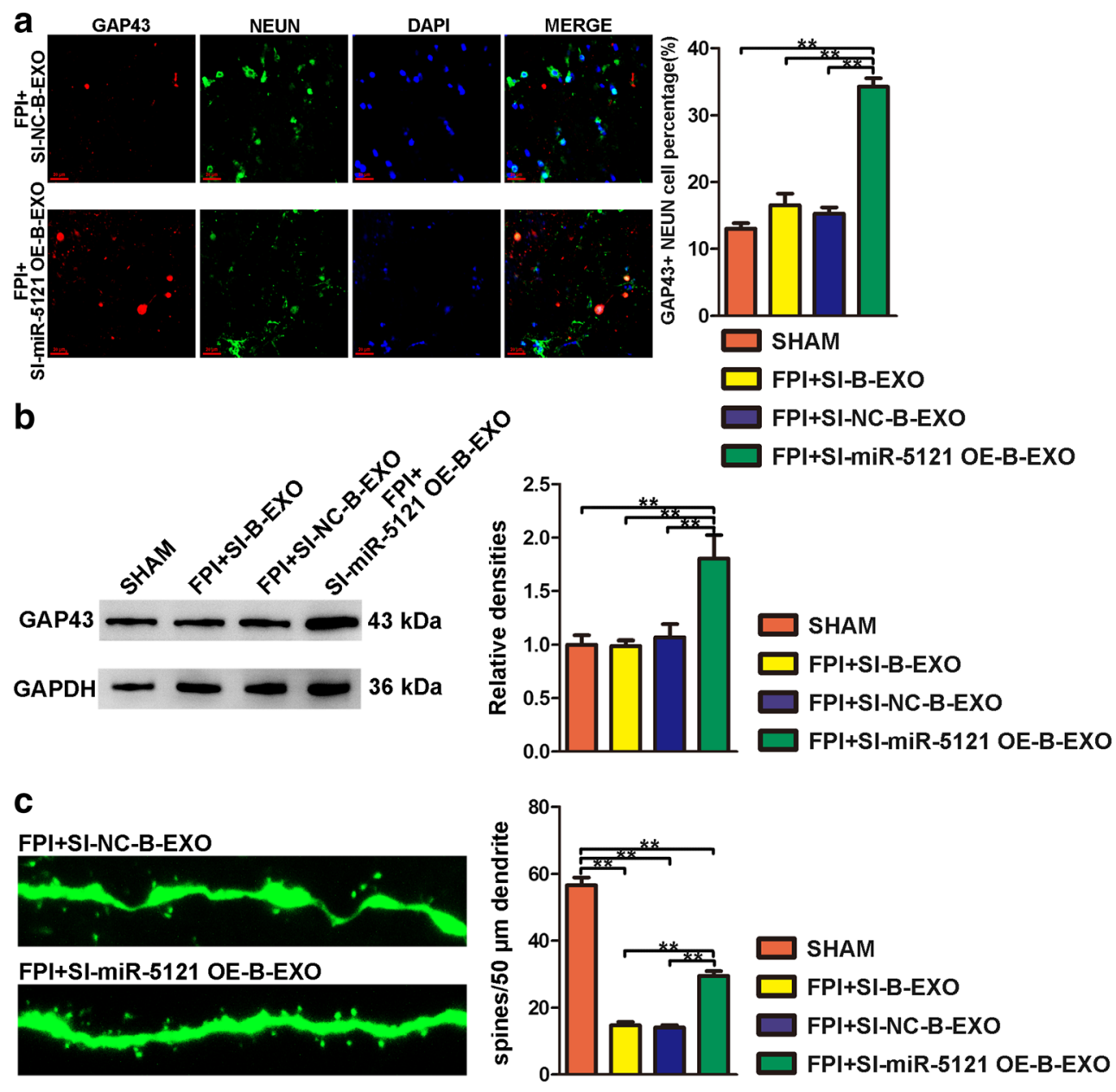

Fig. 8 Tail vein injection of miR-5121 overexpressed exosomes derived from stretch-injured BV2 microglia promoted neurite outgrowth and synapse recovery in vivo. (A) Detection of GAP43 expression in neurons by immunofluorescence. Quantification revealed that tail vein injection of miR-5121 overexpressed exosomes derived from stretch-injured BV2 microglia increased the numbers of GAP43+ neurons. Neurons were identified by NEUN (green). (B) Representative immunoblots of GAP43 in the SHAM group, FPI+SI-B-EXO group, FPI+SI-NC-BEXO group, and FPI+SI-miR-5121 OE-B-EXO group. Quantification revealed that tail vein injection of miR-5121 overexpressed exosomes

significantly decreased in the SI-NEU+SI-B-EXO group, SINEU+SI-NC-B-EXO group, and SI-NEU+SI-miR-5121 OEB-EXO group compared with the CON-NEU group. In addition, a Sholl analysis of the neurons was performed to evaluate the changes in dendrite complexity. As shown in Fig. 7(F), the dendrites of SI-miR-5121 OE-B-EXO-treated neurons showed more intersections than those of SI-B-EXO-treated neurons and SI-NC-B-EXO-treated neurons at $60 \mu \mathrm{m}$ from the neuron soma. Moreover, the dendrites of CON-B-EXOtreated neurons showed more intersections than those of SImiR-5121 OE-B-EXO-treated neurons between 40 and $240 \mu \mathrm{m}$ and at 280 and $340 \mu \mathrm{m}$ from the neuron soma.

These results revealed that elevated exosomal miR-5121 promoted neurite outgrowth and synapse recovery in vitro.
To determine whether elevated exosomal miR-5121 also promoted neurite outgrowth and synapse recovery in vivo, Thy1-GFP knock-in mice were intravenously injected with exosomes isolated from stretch-injured BV2 microglia, negative control stretch-injured BV2 microglia, or miR-5121 overexpressed stretch-injured BV2 microglia through tail vein injection following FPI. As shown in Fig. 8(A), GAP43+ NEUN cell percentage in the pericontusion region was significantly increased in the FPI+SI-miR-5121 OE-B-EXO group compared with that of the FPI+SI-B-EXO group and FPI+SINC-B-EXO group 1 day following FPI. In addition, we also detected the expression level of GAP43 in the pericontusion region by western blot. As shown in Fig. 8(B), the expression level of GAP43 was significantly increased in the FPI+SI- 
Fig. 9 Tail vein injection of miR5121 overexpressed exosomes derived from stretch-injured BV2 microglia improved motor coordination in mice after FPI. (A) Latency and (B) percentage of foot-slips in the beam walking test at 1 and 3 days following FPI were measured. Quantification revealed that mice in the FPI+SImiR-5121 OE-B-EXO group crossed the beams significantly faster than mice in the FPI+SI-BEXO group and FPI+SI-NC-BEXO group in both $3-\mathrm{cm}, 2-\mathrm{cm}$, and $1-\mathrm{cm}$ width trials at 1 day following FPI and only in the 1$\mathrm{cm}$ width trial at 3 days following FPI. Mice in the FPI+SI-miR5121 OE-B-EXO group showed significant decreases in the percentage of foot-slips compared with mice in the FPI+SI-B-EXO group and FPI+SI-NC-B-EXO group in 2-cm and 1-cm width trials at both 1 day and 3 days following FPI. All data are represented as the mean \pm SEM; $n=7$; two-way repeated measures ANOVA with Bonferroni post hoc test was used to analyze significant differences. $* p<0.05$, $* * p<0.01$
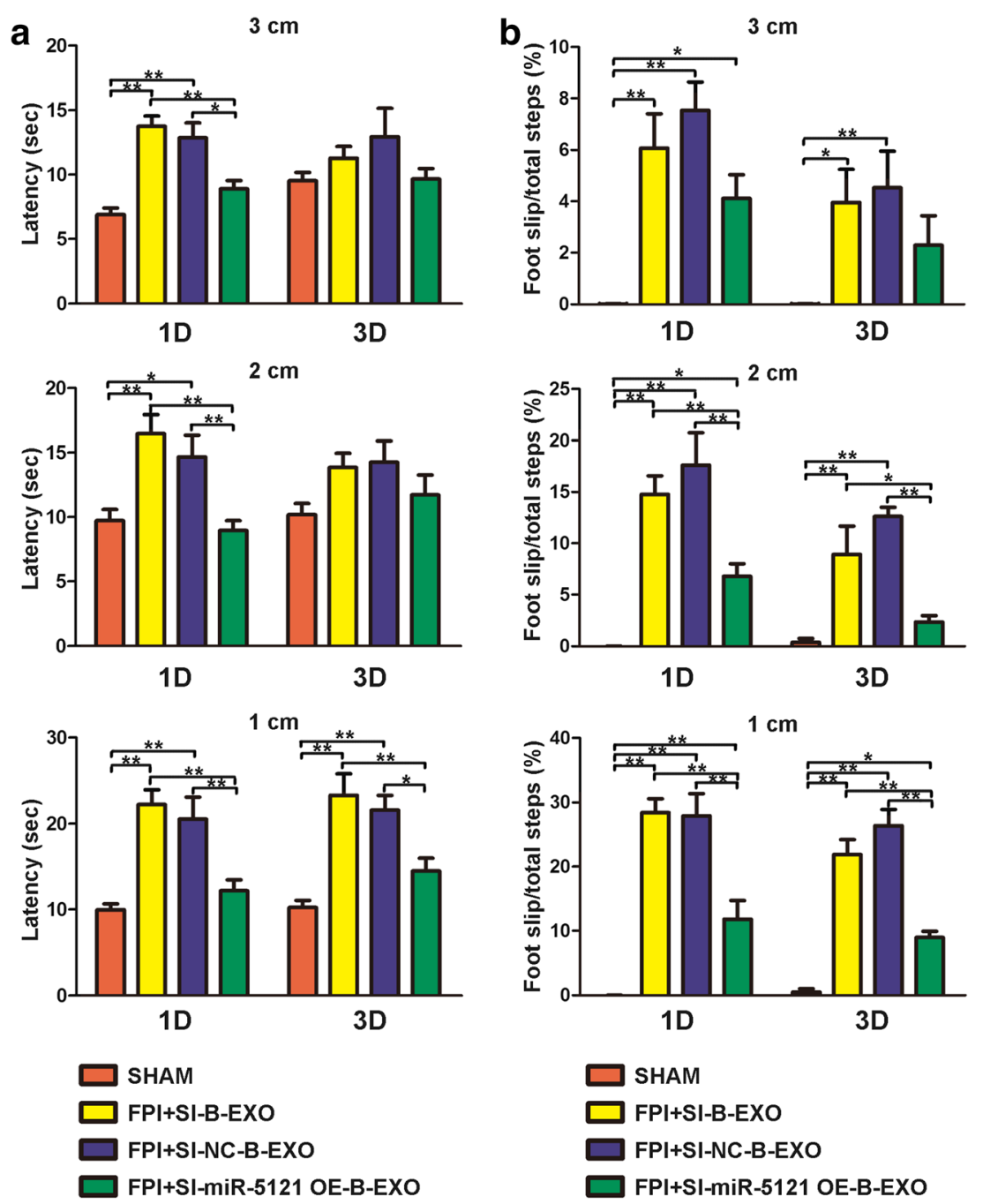

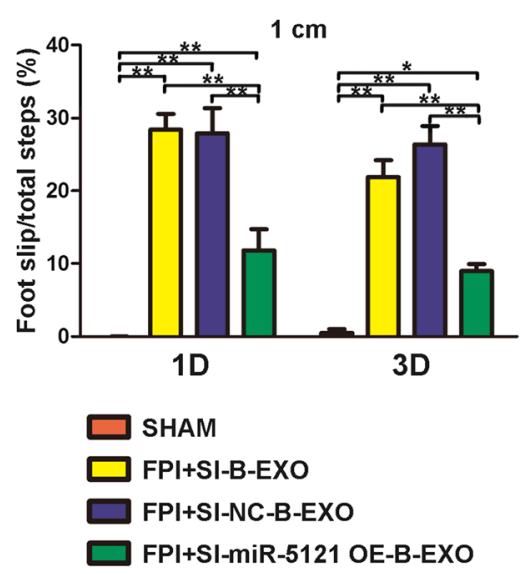

miR-5121 OE-B-EXO group compared with that of the FPI+ SI-B-EXO group and FPI+SI-NC-B-EXO group. Furthermore, spine quantification showed that apical dendritic spine density in the pericontusion region was also significantly increased in the FPI+SI-miR-5121 OE-B-EXO group compared with that of the FPI+SI-B-EXO group and FPI+SI-NCB-EXO group 1 day following FPI (Fig. 8(C)). However, the apical dendritic spine density in the FPI+SI-miR-5121 OE-BEXO group still did not reach the normal level.

These results revealed that elevated exosomal miR-5121 also promoted neurite outgrowth and synapse recovery in vivo.

\section{Elevated Exosomal miR-5121 Improved Motor Coordination in Mice After FPI}

To determine whether elevated exosomal miR-5121 improved neurological functions, the beam walking test was performed to evaluate the motor coordination and balance of the mice. At 1 day after FPI, SI-miR-5121 OE-B-EXO-treated mice performed a significant decrease in time and percentage of foot- slips when they crossed the $2 \mathrm{~cm}$ and $1 \mathrm{~cm}$ width beams in comparison to SI-B-EXO-treated and SI-NC-B-EXO-treated mice (Fig. 9(A, B)). However, for the 3-cm width beam, SImiR-5121 OE-B-EXO-treated mice only performed a significant decrease in time to cross the beam and showed no significant difference in percentage of foot-slips compared with SIB-EXO-treated and SI-NC-B-EXO-treated mice.

At 3 days after FPI, SI-miR-5121 OE-B-EXO-treated mice performed a significant decrease in time and percentage of foot-slips when they crossed the 1-cm width beam in comparison to SI-B-EXO-treated and SI-NC-B-EXO-treated mice (Fig. 9(A, B)). For the 2-cm width beam, SI-miR-5121 OEB-EXO-treated mice only performed a significant decrease in percentage of foot-slips to cross the beam and showed no significant difference in time compared with SI-B-EXOtreated and SI-NC-B-EXO-treated mice. However, there was no significant difference among SI-B-EXO-treated, SI-NC-BEXO-treated, and SI-miR-5121 OE-B-EXO-treated mice in time and percentage of foot-slips to cross the $3-\mathrm{cm}$ width beam. 
These results revealed that elevated exosomal miR-5121 improved neurological functions of the mice after FPI.

\section{Exosomal miR-5121 Might Promote Neurite Outgrowth and Synapse Recovery by Regulation the Expression of its Downstream Target RGMa}

To further explore the underlying mechanism that miR5121 promoted neurite outgrowth and synapse recovery, we performed target prediction search of miR-5121 through miRBase database. The predicted target genes which may be associated with neurite outgrowth and synapse recovery were identified by GO analysis (Fig. S4 and Table S2) and KEGG pathway analysis (Fig. S5 and Table S3). Among these genes, we chose RGMa (the upstream activator of RhoA-Rho kinase pathway) for further study. We detected the expression levels of RGMa and its downstream effector RhoA-GTP in the pericontusion region of SI-B-EXO-treated, SI-NC-B-EXO-treated, and SImiR-5121 OE-B-EXO-treated mice after FPI by western blot assay. As shown in Fig. 10(A, B), the expression levels of RGMa and RhoA-GTP were significantly decreased in the pericontusion region of SI-miR-5121 OEB-EXO-treated mice compared with that of SI-B-EXOtreated and SI-NC-B-EXO-treated mice at 1 day after FPI. Furthermore, the dual-luciferase reporter assay demonstrated that miR-5121 mimic significantly inhibited the luciferase activity in $293 \mathrm{~T}$ cells transfected with the pmirGLO-RGMa-3'UTR-WT reporter, but not in 293T cells transfected with the pmirGLO-RGMa-3'UTR-MUT reporter (Fig. 10(C, D)). The result suggested that RGMa was the direct target of miR-5121.

To investigate the effect of RhoA-Rho kinase pathway on neurite outgrowth and synapse recovery after TBI, we detected the expression levels of GAP43, PSD-95, GluR1, and Synaptophysin in the pericontusion region of SI-B-EXO-treated, SI-B-EXO+Saline-treated, and SIB-EXO+Y-27632-treated mice after FPI by western blot assay. As shown in Fig. 10(E, F), Rho kinase inhibitor, Y-27632, significantly increased the protein levels of GAP43, PSD-95, GluR1, and Synaptophysin in the pericontusion region compared with the FPI+SI-B-EXO group and FPI+SI-B-EXO+Saline group. These results revealed that inhibition of RhoA-Rho kinase pathway by specific Rho kinase inhibitor could reverse the suppression effect of stretch-injured microglia-derived exosomes on neurite outgrowth and synapse recovery after TBI.

Together, these results demonstrated that exosomal miR5121 might promote neurite outgrowth and synapse recovery by regulation of the expression of its downstream target RGMa.

\section{Discussion}

Microglia play a critical role in regulating synaptic plasticity, axon outgrowth, and neuronal death in physiological condition [11-13]. However, in the acute stage following TBI, activated microglia aggravate neuron death and impede neurite outgrowth and synapse recovery [15-17]. The underlying mechanism has not been clearly elucidated. In the present study, our result revealed that stretch-injured microglia may suppress neurite outgrowth and synapse recovery partially through downregulating exosomal miR-5121 delivered to stretch-injured neurons after TBI.

Exosomes are extracellular vesicles of $40-150 \mathrm{~nm}$ in diameter secreted by most cell types in CNS including microglia, astrocyte, and neurons $[30,31]$. These exosomes encapsulate a range of biological molecules including mRNAs, microRNA, and proteins, and participate in intercellular crosstalk in both physiological and pathological conditions [32]. Recent studies have revealed that microglia-derived exosomes play a critical role in microglia-neuron interaction in healthy and pathological brains. In physiological condition, microglia-derived exosomes can exhibit a strong interaction with neurites and significantly increase neurite outgrowth [33, 34]. However, in neurodegenerative disease, such as Alzheimer's disease and Parkinson's disease, exosomes derived from activated microglia deliver misfolded proteins to neurons which cause neuronal dysfunction and death $[35,36]$.

In the present study, we used a microglia stretch-injured model to investigate the effect of microglia-derived exosomes on neurons after TBI. Several previous studies reported that increasing degrees of stretch-induced injury produced increased cell injury [37, 38]. Consistent with these studies, we found severe stretch-induced injury could cause significant microglia cell apoptosis, which might be associated with the M1 polarization of microglia after severe stretch-induced injury. Compared with the conventional LPS-induced M1-type microglia, stretch-injury-induced M1-type microglia released much less M1-type cytokines, suggestion that LPS induced a higher degree of microglia activation.

A recent study reported that stimulation with LPS could significantly increase microvesicle release in microglia [39]. In the present study, we also found that severe stretch-induced injury could increase exosome release in microglia. The reason behind this increase may be associated with $\mathrm{P} 2 \mathrm{X}_{7}$ receptor activation. $\mathrm{P} 2 \mathrm{X}_{7}$ receptor is a member of the purinergic receptor family that predominantly presents on microglia [40]. Several researches revealed that MV shedding and exosome release were activated by $\mathrm{P} 2 \mathrm{X}_{7}$ receptor in microglial cells [41, 42]. Moreover, $\mathrm{P} 2 \mathrm{X}_{7}$ receptor activity is significantly increased after microglia activation [43].

In order to investigate the effect of microglia-derived exosomes on neurons after TBI, neurons were co-cultured with microglia-derived exosomes. Our results revealed that 

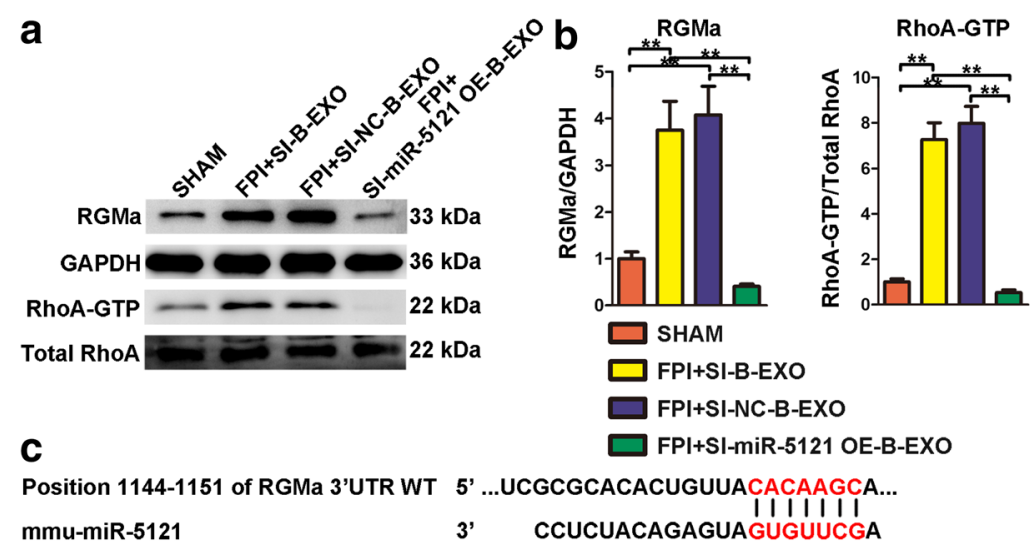

$$
\begin{aligned}
& \text { Position 1144-1151 of RGMa 3'UTR WT 5' ...UCGCGCACACUGUUACACAAGCA... } \\
& \text { Position 1144-1151 of RGMa 3'UTR MUT 5' ...UCGCGCACACUGUUAGUGUUCGA... }
\end{aligned}
$$

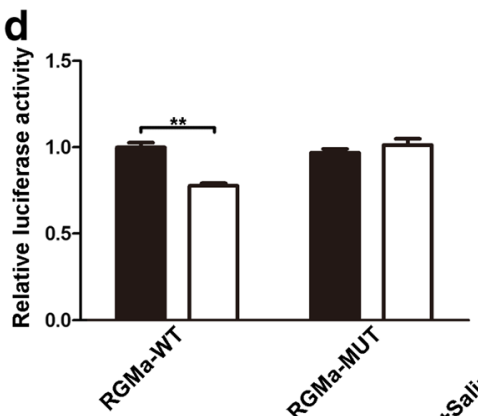

e

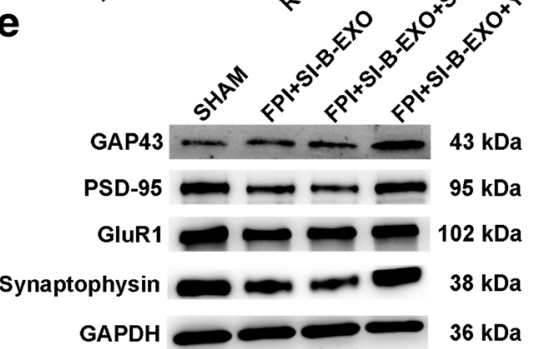

Fig. 10 Exosomal miR-5121 targeted 3'UTR of RGMa. (A) Representative immunoblots of RGMa, RhoA-GTP, and total RhoA in the SHAM group, FPI+SI-B-EXO group, FPI+SI-NC-B-EXO group, and FPI+SI-miR-5121 OE-B-EXO group. (B) Quantification revealed that the expression levels of RGMa and RhoA-GTP was significantly decreased in the FPI+SI-miR-5121 OE-B-EXO group compared with that of the FPI+SI-B-EXO group and FPI+SI-NC-B-EXO group. (C) Wildtype and mutation-type of the RGMa 3'UTR binding site of miR-5121. (D) Quantification revealed that miR-5121 mimic reduced the luciferase activity in 293T cells transfected with the pmirGLO-RGMa-3'UTR-WT

stretch-injured BV2 microglia-derived exosomes could significantly decrease the protein levels of GAP43 (a marker used to evaluate neurite sprouting and synapse recovery $[44,45])$, PSD-95 (a postsynaptic marker used to evaluate synapse density and loss [46, 47]), GluR1 (a postsynaptic marker used to evaluate excitatory synapse density [48, 49]), and Synaptophysin (a presynaptic marker $[50,51]$ ) and dendritic complexity in stretch-injured neurons compared with uninjured BV2 microglia-derived exosomes. Interestingly, we

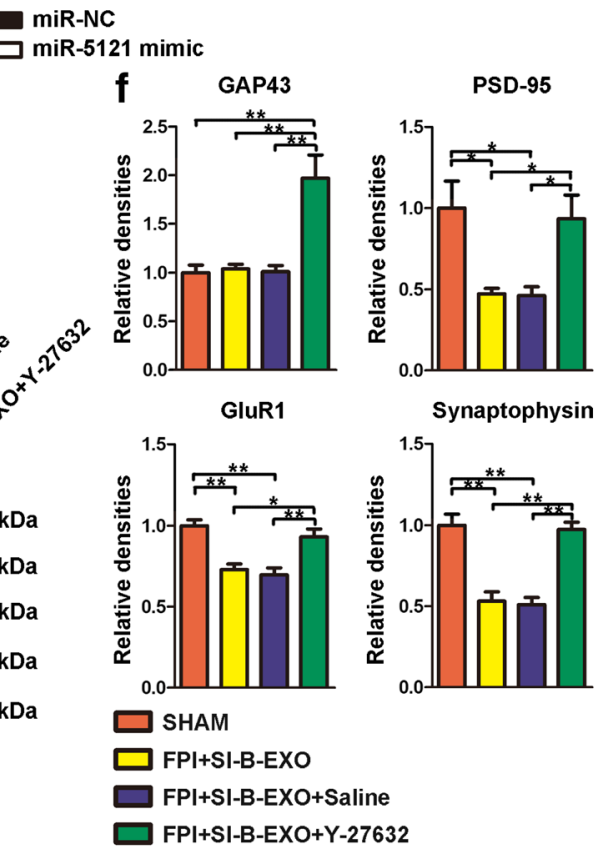

reporter, but not in $293 \mathrm{~T}$ cells transfected with the pmirGLO-RGMa-3' UTR-MUT reporter $(n=3)$. (E) Representative immunoblots of GAP43, PSD-95, GluR1, and Synaptophysin in the SHAM group, FPI+SI-B$\mathrm{EXO}$ group, FPI+SI-B-EXO+Saline group, and FPI+SI-B-EXO+Y27632 group. (F) Quantification revealed that Rho kinase inhibitor, Y-27632, significantly increased the protein levels of GAP43, PSD-95, GluR1, and Synaptophysin in the pericontusion region. All data are represented as the mean $\pm \mathrm{SEM} ; n=6$; one-way ANOVA with Tukey's post hoc test and two-tailed $t$ tests were used to analyze significant differences. $* p<0.05, * * p<0.01$

found that stretch-injured neurons took up more exosomes than uninjured neurons, and neurons took up more exosomes derived from uninjured BV2 microglia than that derived from stretch-injured BV2 microglia. A previous study showed that cells took up extracellular vesicles (EVs) by a variety of endocytic pathways which depended on proteins and glycoproteins expressed on EV and target cell surface [52]. Thus, we hypothesized that this phenomenon might be associated with expression change of proteins and glycoproteins on the 
surface of both the neurons and microglia-derived exosomes after TBI. The exact underlying mechanism might need further investigation.

Furthermore, in order to investigate the role of microgliaderived exosomes in neurite outgrowth and synapse recovery after TBI in vivo, mice that underwent FPI were intravenously injected with PKH26-labeled exosomes derived from microglia by tail vein injection. Several studies reported that exosomes could penetrate blood-brain barrier and act as drug delivery vehicles $[53,54]$. In the present study, we also found microglia-derived exosomes could penetrate the blood-brain barrier and be absorbed by neurons in the pericontusion region. The exosomes derived from stretch-injured microglia could decrease GAP43+ NEUN cell percentage, expression level of GAP43, and apical dendritic spine density in the pericontusion region compared with uninjured microgliaderived exosomes. Here, we noticed that although the apical dendritic spine density was significantly increased in uninjured microglia-derived exosome-treated mice, it still did not reach the normal level. However, in the in vitro experiment, the protein levels of PSD-95, GluR1, and Synaptophysin showed no significant difference between the CON-NEU group and SI-NEU+CON-B-EXO group. We hypothesized that exosomes might be taken up by other organs before penetrating the blood-brain barrier [55] and taken up by other cell types besides neurons after penetrating the blood-brain barrier as reported before [56]. Thus, injured neurons in the pericontusion region did not take up enough doses of exosomes. Furthermore, the beam walking test revealed that CON-B-EXO treatment significantly improved the motor coordination function of TBI mice. These findings further confirmed that CON-B-EXO derived from uninjured microglia could exert a protective effect in injured brains after TBI.

In a recent study in mice, Harrison and colleagues demonstrated that the expression of five microRNAs (miR-212, miR21, miR-146, miR-7a, and miR-7b) was significantly changed in EVs isolated from cortical homogenates following a controlled cortical impact injury, suggesting that TBI induced a significant change in EV-associated miRNA in injured brain [57]. Based on these findings, further research reported that elevated exosomal miR-21-5p derived from scratch-injured PC12 cells could promote the M1 polarization of microglia and further inhibit neurite outgrowth of PC12 cells [58]. To determine whether exosomal microRNAs derived from stretch-injured BV2 microglia were involved in neurite outgrowth and synapse recovery suppression after TBI, we performed microRNA microarray analysis to investigate the expression profile change of exosomal microRNAs derived from microglia after stretch-induced injury. Our microRNA microarray data showed that the expression level of exosomal miR-5121 was decreased most greatly after stretch-induced injury of BV2 microglia. Currently, the study focusing on the role of miR-5121 in neurons is still lacking. Further GO analysis and pathway analysis of predicted target genes of miR-5121 showed that miR-5121 might be associated with neurite outgrowth and synapse recovery of neurons (GO analysis: negative regulation of axonogenesis; axon guidance; regulation of synapse assembly and regulation of synapse structure and activity. Pathway analysis: axon guidance and longterm depression). Consistent with the GO analysis and pathway analysis, our following experiments demonstrated that elevated exosomal miR-5121 derived from stretch-injured BV2 microglia could promote neurite outgrowth and synapse recovery in vitro and in vivo. Following beam walking test revealed that elevated exosomal miR-5121 improved neurological functions of the mice after FPI.

To further explore the underlying mechanism that miR5121 promoted neurite outgrowth and synapse recovery, predicted miR-5121 targets were grouped according to GO analysis and pathway analysis. Potential downstream targets of miR-5121 related to neurite outgrowth and synapse recovery were selected. Among these potential targets, we chose RGMa (GO analysis: negative regulation of axonogenesis and negative regulation of neuron projection development) for further study. RGMa is a membrane-bound protein that regulates axon guidance, synapse formation, neuronal differentiation, and neuronal cell death by binding to its receptor Neogenin and activation of its downstream RhoA-Rho kinase pathway [59-61]. The expression level of RGMa is significantly increased following brain ischemia or spinal cord injury [62, 63]. Inhibition of RGMa promotes neurite outgrowth, synapse formation, and neural function recovery in rat after brain ischemia or spinal cord injury [60, 64-66]. In the present study, our results demonstrated that the expression levels of RGMa and its downstream effector RhoA-GTP in the pericontusion region of SI-miR-5121 OE-B-EXO-treated mice were significantly decreased compared to that of SI-B-EXO-treated and SI-NC-B-EXO-treated mice after FPI. Moreover, the dualluciferase reporter assay confirmed that RGMa was the direct target of miR-5121. Furthermore, treatment of Rho kinase inhibitor, Y-27632, significantly increased the protein levels of GAP43, PSD-95, GluR1, and Synaptophysin in the pericontusion region compared with the FPI+SI-B-EXO group and FPI+SI-B-EXO+Saline group at 1 day after FPI, suggesting that RhoA-Rho kinase pathway played a critical role in neurite outgrowth and synapse recovery after TBI. Thus, exosomal miR-5121 may promote neurite sprouting and synapse recovery through direct targeting RGMa.

Although our results demonstrated that decreased level of exosomal miR-5121 derived from microglia after stretchinduced injury might suppress neurite outgrowth and synapse recovery through targeting RGMa, it is worthy of note that the protein levels of GAP43, PSD-95, and GluR1 were significantly decreased in the SI-NEU+SI-B-EXO group compared with that of the SI-NEU group. These results revealed that other biological molecules encapsulated in stretch-injured 
microglia-derived exosomes might also participate in suppression of neurite outgrowth and synapse recovery. Our microarray data showed that there were a total of 7 microRNAs that were upregulated (fold change $>3$ ) and 4 microRNAs that were downregulated (fold change $>3$ ) in exosomes derived from stretch-injured BV2 microglia compared with that of uninjured BV2 microglia-derived exosomes. Some of them may be involved in regulation of neurite outgrowth and synapse recovery. Thus, further studies are warranted to investigate whether other biological molecules in microglia-derived exosomes are involved in regulation of neurite outgrowth and synapse recovery after TBI.

In summary, our present study demonstrates that injured microglia may suppress neurite outgrowth and synapse recovery partially through downregulating exosomal miR-5121 delivered to injured neurons after TBI. Further studies are needed to verify if targeting microglia-derived exosomes can be used as a novel strategy for clinical TBI treatment.

Supplementary Information The online version contains supplementary material available at https://doi.org/10.1007/s13311-020-00999-z.

Acknowledgements This research was supported by grants from the National Natural Science Foundation of China (81772685), the Natural Science Foundation of Guangdong Province (2018A030310042), and the Guangdong Basic and Applied Basic Research Foundation (2019A1515011241).

Required Author Forms Disclosure forms provided by the authors are available with the online version of this article.

Authors' Contributions $\mathrm{C}-\mathrm{C} \mathrm{Z}$ and $\mathrm{C}-\mathrm{F}$ W carried out the experiments and contributed to study design and acquisition of data; $\mathrm{Y} H$ contributed to data analysis and manuscript preparation; X-J H contributed to data analysis; Y-F D contributed to study design, experiment supervision, and final approval of the manuscript; W-P L contributed to technical support and experiment advise.

\section{Compliance with Ethical Standards}

Conflict of Interest The authors declare that they have no conflict of interest.

\section{References}

1. Popescu C, Anghelescu A, Daia C, Onose G. Actual data on epidemiological evolution and prevention endeavours regarding traumatic brain injury. Journal of medicine and life. 2015;8(3):272-277.

2. Jiang JY, Gao GY, Feng JF, et al. Traumatic brain injury in China. The Lancet Neurology. 2019;18(3):286-295.

3. Rosenfeld JV, Maas AI, Bragge P, Morganti-Kossmann MC, Manley GT, Gruen RL. Early management of severe traumatic brain injury. Lancet. 2012;380(9847):1088-1098.

4. Gruenbaum SE, Zlotnik A, Gruenbaum BF, Hersey D, Bilotta F. Pharmacologic Neuroprotection for Functional Outcomes After Traumatic Brain Injury: A Systematic Review of the Clinical Literature. CNS drugs. 2016;30(9):791-806.
5. Tsitsopoulos PP, Abu Hamdeh S, Marklund N. Current Opportunities for Clinical Monitoring of Axonal Pathology in Traumatic Brain Injury. Frontiers in neurology. 2017;8:599.

6. Winston CN, Chellappa D, Wilkins T, et al. Controlled cortical impact results in an extensive loss of dendritic spines that is not mediated by injury-induced amyloid-beta accumulation. Journal of neurotrauma. 2013;30(23):1966-1972.

7. Jin D, Liu Y, Sun F, Wang X, Liu X, He Z. Restoration of skilled locomotion by sprouting corticospinal axons induced by codeletion of PTEN and SOCS3. Nature communications. 2015;6: 8074.

8. Deng B, Li L, Gou X, et al. TAT-PEP Enhanced Neurobehavioral Functional Recovery by Facilitating Axonal Regeneration and Corticospinal Tract Projection After Stroke. Molecular neurobiology. 2018;55(1):652-667.

9. Starkey ML, Bartus K, Barritt AW, Bradbury EJ. Chondroitinase $\mathrm{ABC}$ promotes compensatory sprouting of the intact corticospinal tract and recovery of forelimb function following unilateral pyramidotomy in adult mice. The European journal of neuroscience. 2012;36(12):3665-3678.

10. Xavier AL, Menezes JR, Goldman SA, Nedergaard M. Fine-tuning the central nervous system: microglial modelling of cells and synapses. Philosophical transactions of the Royal Society of London Series B, Biological sciences. 2014;369(1654):20130593.

11. Tremblay ME, Stevens B, Sierra A, Wake H, Bessis A, Nimmerjahn A. The role of microglia in the healthy brain. The Journal of neuroscience : the official journal of the Society for Neuroscience. 2011;31(45):16064-16069.

12. Salter MW, Beggs S. Sublime microglia: expanding roles for the guardians of the CNS. Cell. 2014;158(1):15-24.

13. Arnoux I, Audinat E. Fractalkine Signaling and Microglia Functions in the Developing Brain. Neural plasticity. 2015;2015: 689404.

14. Fourgeaud L, Traves PG, Tufail Y, et al. TAM receptors regulate multiple features of microglial physiology. Nature. 2016;532(7598):240-244

15. Izzy S, Liu Q, Fang Z, et al. Time-Dependent Changes in Microglia Transcriptional Networks Following Traumatic Brain Injury. Frontiers in cellular neuroscience. 2019;13:307.

16. Madathil SK, Wilfred BS, Urankar SE, et al. Early Microglial Activation Following Closed-Head Concussive Injury Is Dominated by Pro-Inflammatory M-1 Type. Frontiers in neurology. 2018;9:964

17. Wang CF, Zhao CC, Liu WL, et al. Depletion of Microglia Attenuates Dendritic Spine Loss and Neuronal Apoptosis in the Acute Stage of Moderate Traumatic Brain Injury in Mice. Journal of neurotrauma. 2020;37(1):43-54

18. Koritzinsky EH, Street JM, Star RA, Yuen PS. Quantification of Exosomes. Journal of cellular physiology. 2017;232(7):1587-1590.

19. Bang C, Thum T. Exosomes: new players in cell-cell communication. The international journal of biochemistry \& cell biology. 2012;44(11):2060-2064.

20. Huang S, Ge X, Yu J, et al. Increased miR-124-3p in microglial exosomes following traumatic brain injury inhibits neuronal inflammation and contributes to neurite outgrowth via their transfer into neurons. FASEB journal : official publication of the Federation of American Societies for Experimental Biology. 2018;32(1):512528.

21. Salvador E, Neuhaus W, Foerster C. Stretch in brain microvascular endothelial cells (cEND) as an in vitro traumatic brain injury model of the blood brain barrier. Journal of visualized experiments : JoVE. 2013(80):e50928.

22. Wu CH, Hung TH, Chen CC, et al. Post-injury treatment with 7,8 dihydroxyflavone, a TrkB receptor agonist, protects against experimental traumatic brain injury via PI3K/Akt signaling. PloS one. 2014;9(11):e113397. 
23. Lin L, Desai R, Wang X, Lo EH, Xing C. Characteristics of primary rat microglia isolated from mixed cultures using two different methods. Journal of neuroinflammation. 2017;14(1):101.

24. Thery C, Amigorena S, Raposo G, Clayton A. Isolation and characterization of exosomes from cell culture supernatants and biological fluids. Current protocols in cell biology. 2006; Chapter 3:Unit 3 22.

25. Chai RC, Jiang JH, Wong AY, et al. AQP5 is differentially regulated in astrocytes during metabolic and traumatic injuries. Glia. 2013;61(10):1748-1765.

26. Carbonell WS, Maris DO, McCall T, Grady MS. Adaptation of the fluid percussion injury model to the mouse. Journal of neurotrauma. 1998;15(3):217-229.

27. Hu YB, Ren RJ, Zhang YF, et al. Rho-associated coiled-coil kinase 1 activation mediates amyloid precursor protein site-specific Ser655 phosphorylation and triggers amyloid pathology. Aging cell. 2019;18(5):e13001.

28. Zhao CC, Wang CF, Li WP, et al. Mild Hypothermia Promotes Pericontusion Neuronal Sprouting via Suppressing Suppressor of Cytokine Signaling 3 Expression after Moderate Traumatic Brain Injury. Journal of neurotrauma. 2017;34(8):1636-1644.

29. Webster SJ, Bachstetter AD, Van Eldik LJ. Comprehensive behavioral characterization of an APP/PS-1 double knock-in mouse model of Alzheimer's disease. Alzheimer's research \& therapy. 2013;5(3):28.

30. Greening DW, Xu R, Ji H, Tauro BJ, Simpson RJ. A protocol for exosome isolation and characterization: evaluation of ultracentrifugation, density-gradient separation, and immunoaffinity capture methods. Methods in molecular biology. 2015;1295:179-209.

31. Budnik V, Ruiz-Canada C, Wendler F. Extracellular vesicles round off communication in the nervous system. Nature reviews Neuroscience. 2016;17(3):160-172.

32. Kalluri R, LeBleu VS. The biology, function, and biomedical applications of exosomes. Science. 2020;367(6478).

33. Raffo-Romero A, Arab T, Al-Amri IS, et al. Medicinal Leech CNS as a Model for Exosome Studies in the Crosstalk between Microglia and Neurons. International journal of molecular sciences. 2018;19(12)

34. Lemaire Q, Raffo-Romero A, Arab T, et al. Isolation of microgliaderived extracellular vesicles: towards miRNA signatures and neuroprotection. Journal of nanobiotechnology. 2019;17(1):119.

35. DeLeo AM, Ikezu T. Extracellular Vesicle Biology in Alzheimer's Disease and Related Tauopathy. Journal of neuroimmune pharmacology : the official journal of the Society on NeuroImmune Pharmacology. 2018;13(3):292-308.

36. Xia Y, Zhang G, Han C, et al. Microglia as modulators of exosomal alpha-synuclein transmission. Cell death \& disease. 2019;10(3): 174.

37. McKinney JS, Willoughby KA, Liang S, Ellis EF. Stretch-induced injury of cultured neuronal, glial, and endothelial cells. Effect of polyethylene glycol-conjugated superoxide dismutase. Stroke. 1996;27(5):934-940

38. Ellis EF, McKinney JS, Willoughby KA, Liang S, Povlishock JT. A new model for rapid stretch-induced injury of cells in culture: characterization of the model using astrocytes. Journal of neurotrauma. 1995; 12(3):325-339.

39. Kumar A, Stoica BA, Loane DJ, et al. Microglial-derived microparticles mediate neuroinflammation after traumatic brain injury. Journal of neuroinflammation. 2017;14(1):47.

40. Yue N, Huang $\mathrm{H}$, Zhu X, et al. Activation of $\mathrm{P} 2 \mathrm{X} 7$ receptor and NLRP3 inflammasome assembly in hippocampal glial cells mediates chronic stress-induced depressive-like behaviors. Journal of neuroinflammation. 2017;14(1):102.

41. Turola E, Furlan R, Bianco F, Matteoli M, Verderio C. Microglial microvesicle secretion and intercellular signaling. Frontiers in physiology. 2012;3:149.
42. Takenouchi T, Tsukimoto M, Iwamaru Y, et al. Extracellular ATP induces unconventional release of glyceraldehyde-3-phosphate dehydrogenase from microglial cells. Immunology letters. 2015;167(2):116-124.

43. Leduc-Pessah H, Weilinger NL, Fan CY, Burma NE, Thompson RJ, Trang T. Site-Specific Regulation of P2X7 Receptor Function in Microglia Gates Morphine Analgesic Tolerance. The Journal of neuroscience : the official journal of the Society for Neuroscience. 2017;37(42):10154-10172.

44. Harris NG, Mironova YA, Hovda DA, Sutton RL. Pericontusion axon sprouting is spatially and temporally consistent with a growthpermissive environment after traumatic brain injury. Journal of neuropathology and experimental neurology. 2010;69(2):139-154.

45. Stokowska A, Atkins AL, Moran J, et al. Complement peptide C3a stimulates neural plasticity after experimental brain ischaemia. Brain : a journal of neurology. 2017;140(2):353-369.

46. Kincheski GC, Valentim IS, Clarke JR, et al. Chronic sleep restriction promotes brain inflammation and synapse loss, and potentiates memory impairment induced by amyloid-beta oligomers in mice. Brain, behavior, and immunity. 2017;64:140-151.

47. Irie M, Hata $\mathrm{Y}$, Takeuchi $\mathrm{M}$, et al. Binding of neuroligins to PSD95. Science. 1997;277(5331):1511-1515.

48. Hollmann M, Heinemann S. Cloned glutamate receptors. Annual review of neuroscience. 1994;17:31-108.

49. Liu J, Pasini S, Shelanski ML, Greene LA. Activating transcription factor 4 (ATF4) modulates post-synaptic development and dendritic spine morphology. Frontiers in cellular neuroscience. 2014;8: 177.

50. Wiedenmann B, Franke WW. Identification and localization of synaptophysin, an integral membrane glycoprotein of $\mathrm{Mr} 38,000$ characteristic of presynaptic vesicles. Cell. 1985;41(3):1017-1028.

51. Kokotos AC, Harper CB, Marland JRK, Smillie KJ, Cousin MA, Gordon SL. Synaptophysin sustains presynaptic performance by preserving vesicular synaptobrevin-II levels. Journal of neurochemistry. 2019;151(1):28-37.

52. Mulcahy LA, Pink RC, Carter DR. Routes and mechanisms of extracellular vesicle uptake. Journal of extracellular vesicles. 2014;3.

53. Jia G, Han Y, An Y, et al. NRP-1 targeted and cargo-loaded exosomes facilitate simultaneous imaging and therapy of glioma in vitro and in vivo. Biomaterials. 2018;178:302-316.

54. Tian T, Zhang HX, He CP, et al. Surface functionalized exosomes as targeted drug delivery vehicles for cerebral ischemia therapy. Biomaterials. 2018;150:137-149.

55. Song Y, Li Z, He T, et al. M2 microglia-derived exosomes protect the mouse brain from ischemia-reperfusion injury via exosomal miR-124. Theranostics. 2019;9(10):2910-2923.

56. Ge X, Guo M, Hu T, et al. Increased Microglial Exosomal miR124-3p Alleviates Neurodegeneration and Improves Cognitive Outcome after rmTBI. Molecular therapy : the journal of the American Society of Gene Therapy. 2020;28(2):503-522.

57. Harrison EB, Hochfelder CG, Lamberty BG, et al. Traumatic brain injury increases levels of miR-21 in extracellular vesicles: implications for neuroinflammation. FEBS open bio. 2016;6(8):835-846.

58. Yin Z, Han Z, Hu T, et al. Neuron-derived exosomes with high miR-21-5p expression promoted polarization of M1 microglia in culture. Brain, behavior, and immunity. 2020;83:270-282.

59. De Vries M, Cooper HM. Emerging roles for neogenin and its ligands in CNS development. Journal of neurochemistry. 2008;106(4):1483-1492.

60. Kyoto A, Hata K, Yamashita T. Synapse formation of the corticospinal axons is enhanced by RGMa inhibition after spinal cord injury. Brain research. 2007;1186:74-86.

61. Conrad S, Genth H, Hofmann F, Just I, Skutella T. NeogeninRGMa signaling at the growth cone is bone morphogenetic 
protein-independent and involves RhoA, ROCK, and PKC. The Journal of biological chemistry. 2007;282(22):16423-16433.

62. Li M, Wen Y, Zhang R, Xie F, Zhang G, Qin X. Adenoviral vectorinduced silencing of RGMa attenuates blood-brain barrier dysfunction in a rat model of MCAO/reperfusion. Brain research bulletin. 2018;142:54-62.

63. Schwab JM, Conrad S, Monnier PP, Julien S, Mueller BK, Schluesener HJ. Spinal cord injury-induced lesional expression of the repulsive guidance molecule (RGM). The European journal of neuroscience. 2005;21(6):1569-1576.

64. Tao T, Xu G, Si Chen C, Feng J, Kong Y, Qin X. Minocycline promotes axonal regeneration through suppression of RGMa in rat MCAO/reperfusion model. Synapse. 2013;67(4):189-198.
65. Jiang F, Yin H, Qin X. Fastigial nucleus electrostimulation reduces the expression of repulsive guidance molecule, improves axonal growth following focal cerebral ischemia. Neurochemical research. 2012;37(9):1906-1914.

66. Mothe AJ, Coelho M, Huang L, et al. Delayed administration of the human anti-RGMa monoclonal antibody elezanumab promotes functional recovery including spontaneous voiding after spinal cord injury in rats. Neurobiology of disease. 2020;143:104995.

Publisher's Note Springer Nature remains neutral with regard to jurisdictional claims in published maps and institutional affiliations. 